\title{
Theta Functions, Modular Invariance, and Strings
}

\author{
Luis Alvarez-Gaumé, Gregory Moore, and Cumrun Vafa \\ Department of Physics, Harvard University, Cambridge, MA 02138, USA
}

\begin{abstract}
We use Quillen's theorem and algebraic geometry to investigate the modular transformation properties of some quantities of interest in string theory. In particular, we show that the spin structure dependence of the chiral Dirac determinant on a Riemann surface is given by Riemann's theta function. We use this result to investigate the modular invariance of multiloop heterotic string amplitudes.
\end{abstract}

\section{Introduction}

Two-dimensional quantum field theories have served as toy models in attempts to understand more complicated four-dimensional theories. The two dimensional theories capture many essential features of higher dimensions, without sharing the complexities of higher dimensions. Certain features of $2 d$ QFT's such as BoseFermi equivalence have led to a large number of exactly solvable theories.

In string theories, two-dimensional conformal QFT plays an even more important role [1-3]. The string sweeps out a surface as it moves through spacetime, and therefore, the first quantized theory corresponds to a two dimensional QFT. The string can sweep out a surface with any number of handles. Whereas in most well known results one considers the underlying space to be $R^{2}$ (or $R \times S^{1}$, or $S^{1} \times S^{1}$ corresponding to periodic boundary conditions in space or time), for string theories one must consider the space to be an arbitrary Riemann surface. Thus, understanding multiloop string amplitudes requires an understanding of QFT on a Riemann surface. In the general case, few explicit facts are known.

Many questions remain unanswered in string theory. For instance, how can we prove the vanishing of the cosmological constant in superstring theories? This has been shown explicitly at 1-loop. For higher loops, even though there is an indirect argument for the vanishing of the cosmological constant $[3,4]$, one would like to show this important fact more directly.

There are other issues: What happens to the string amplitude in the limit of a degenerating Riemann surface? Are superstring theories finite? Is the perturbation 
expansion valid? Can one develop an operator formalism for surfaces with more than one handle? How does the bosonization work at higher loops? etc.

Motivated by these questions, we address below some issues regarding the determinant of some operators, in particular the chiral Dirac operator, defined on a Riemann surface. We will discuss in some detail the spin structure dependence of the determinant of the Dirac operator. Our main tools are Quillen's holomorphic anomaly, algebraic geometry, and bosonization. We feel that the algebraic geometric techniques are very powerful and well suited for applications to a better understanding of string theories. This viewpoint has also been advocated recently by several groups.

In Sect. 2 we illustrate some of the issues which will be discussed in this paper, in the context of the simple case of the torus. In Sect. 3 we discuss the mathematical concepts which will be used. Many of them, for example, modular transformations, generalized theta functions and spin structures appear as natural generalizations of the concepts already appearing in the case of the torus. Yet others, such as divisors of theta functions and their relation to spin structures, although important for higher loops, do not play a central role in the genus one case.

In Sect. 4 we discuss Quillen's holomorphic anomaly, and a slight generalization of it. This anomaly is the obstruction to expressing as a holomorphic function the determinant of a family of operators depending holomorphically on some complex parameter.

In Sect. 5 we show that the spin dependence of the determinant of the chiral Dirac operator is given by theta functions. In Sect. 6 we discuss these results from the viewpoint of bosonization. In Sect. 7 we apply the results obtained to address some questions related to the modular invariance of the measure for heterotic strings [5]. Finally, in Sect. 8 we present our conclusions.

\section{Fermions on a Torus}

In this section we will consider fermions on a torus. We label the points of the torus by the complex quantity $\sigma_{1}+\tau \sigma_{2}$, where $\sigma_{1}$ and $\sigma_{2}$ are periodic variables with period $2 \pi$. This means that

$$
x \sim x+2 \pi \sim x+2 \pi \tau .
$$

For bosonic fields defined on the torus one requires the periodic boundary conditions:

$$
X\left(\sigma_{1}+2 \pi, \sigma_{2}\right)=X\left(\sigma_{1}, \sigma_{2}+2 \pi\right)=X\left(\sigma_{1}, \sigma_{2}\right) .
$$

For fermions, we have the option of choosing periodic or antiperiodic boundary conditions in each direction. Therefore we have four different possible boundary conditions: $(A, A),(P, A),(A, P)$, and $(P, P)$, where $P(A)$ stands for periodic (antiperiodic). These are called the spin structures. So for genus 1 we have four different spin structures.

For the torus we have chosen $2 \pi$ and $2 \pi \tau$ to generate the lattice defining it. We could have chosen a different basis for the same lattice, thereby obtaining the same torus. For example the tori defined by $2 \pi(1, \tau)$ and $2 \pi(1, \tau+1)$ differ from one another by a global diffeomorphism. To see this we simply cut the torus along the 
$\sigma_{1}$ cycle and rotate the two boundary circles relative to one another by $2 \pi$ and glue them back together. (This is called a Dehn twist, and its general form will be discussed in Sect. 3.)

Different choices of the lattice defining the torus could be obtained by means of $2 \times 2$, invertible (unit determinant) and integral matrices acting on a basis. This is the group $S L(2, Z)$ which is referred to as the modular group. Under an element of $S L(2, Z)$, with entries $a, b, c, d$,

$$
\tau \rightarrow \frac{a \tau+b}{c \tau+d}
$$

The Dehn twists about the $\sigma_{1}$ and $\sigma_{2}$ cycles correspond respectively to

$$
\left(\begin{array}{ll}
1 & 1 \\
0 & 1
\end{array}\right)\left(\begin{array}{ll}
1 & 0 \\
1 & 1
\end{array}\right)
$$

and they generate $S L(2, Z)$.

Under $\operatorname{SL}(2, Z)$ it is easy to see that three spin structures mix, in the sense that

$$
(P, A)_{\tau}=(A, A)_{\tau+1}=(A, P)_{-1 / \tau} .
$$

The $(P, P)$ spin structure is invariant under the action of $S L(2, Z)$. So in a sense we have only two inequivalent spin structures. There is a simple way to characterize each class. We consider the Dirac operator for each of the spin structures which we label by $\alpha$. Let $n(\alpha)$ denote the number of zero modes of the Dirac operator for spin structure $\alpha$. Since the Dirac operator is real, there is no chiral index in two dimensions and therefore $n(\alpha)$ is even. We call a spin structure even (odd) if $\frac{1}{2} n(\alpha)$ is even (odd). It is easy to see in our example of the torus that there are three even spin structures [with $n(\alpha)=0](A, P),(P, A),(A, A)$ and one odd spin structure [with $n(\alpha)=2](P, P)$. Under the action of global diffeomorphisms the two different classes of spin structures cannot mix because they have different number of zero modes for the Dirac operator. In fact more is true: All the spin structures in each class mix under the action of global diffeomorphisms. This is true in the above example and, as we will discuss, it continues to be true for higher genus Riemann surfaces.

Modular transformations are important for string theories. In a string theory we have to divide the path integral measure by the action of diffeomorphisms, and this includes global diffeomorphisms which cannot be reached from identity. Therefore it is necessary for the path integral measure to be invariant under global diffeomorphisms, i.e., modular transformations (For each spin structure it should be invariant under diffeomorphisms preserving that spin structure.)

Note that each spin structure is simply related to a particular one by changing boundary conditions by \pm 1 . We can in fact continuously interpolate between various spin structures if we consider arbitrary twistings of the boundary conditions by a phase. (For this we should allow the spinor to be complex.) So we get a two parameter family of complex spinors on the torus defined by $\theta, \phi$ :

$$
\begin{aligned}
& \psi\left(\sigma_{1}+2 \pi, \sigma_{2}\right)=-e^{2 \pi i \theta} \psi\left(\sigma_{1}, \sigma_{2}\right), \\
& \psi\left(\sigma_{1}, \sigma_{2}+2 \pi\right)=-e^{-2 \pi i \phi} \psi\left(\sigma_{1}, \sigma_{2}\right) .
\end{aligned}
$$


The four spin structures $(A, A),(P, A),(A, P)$ and $(P, P)$ correspond respectively to the twistings $(\theta, \phi)=(0,0),\left(\frac{1}{2}, 0\right),\left(0, \frac{1}{2}\right)$ and $\left(\frac{1}{2}, \frac{1}{2}\right)$. (Even though we have used the twistings of boundary conditions as a formal technique to interpolate between various spin structures, they in fact appear naturally when considering string amplitudes on orbifolds [6].)

Let us denote by $\operatorname{Det}(\theta, \phi)$ the determinant of the chiral Dirac operator with the above twisted boundary conditions. The Hamiltonian for the twisted fermion can be expressed as

$$
H=\sum_{-\infty}^{+\infty}\left(n+\theta-\frac{1}{2}\right): b_{n+\theta-1 / 2}^{\dagger} b_{n+\theta-1 / 2}:+\left(\frac{\theta^{2}}{2}-\frac{1}{24}\right),
$$

where the constant term comes from normal ordering, and

$$
\begin{aligned}
& \left\{b_{\eta_{1}}^{\dagger}, b_{\eta_{2}}\right\}=\delta_{\eta_{1}, \eta_{2}}, \\
& \left\{b_{\eta_{1}}^{\dagger}, b_{\eta_{2}}^{\dagger}\right\}=\left\{b_{\eta_{1}}, b_{\eta_{2}}\right\}=0 .
\end{aligned}
$$

We define $g$ (up to a phase) by $g b_{n+\theta-1 / 2} g^{-1}=-e^{-2 \pi i \phi} b_{n+\theta-1 / 2}$, and also let $q=e^{2 \pi i \tau}$, then the determinant can be computed up to a phase using the partition function

$\operatorname{Det}(\theta, \phi)=\operatorname{Tr} g q^{H}=e^{2 \pi i \theta \phi} q^{\frac{\theta^{2}}{2}-\frac{1}{24}} \prod_{n=1}^{\infty}\left(1+q^{n+\theta-\frac{1}{2}} e^{2 \pi i \phi}\right)\left(1+q^{n-\theta-\frac{1}{2}} e^{-2 \pi i \phi}\right)$.

(The phase in front has been chosen for convenience.) The argument for the equality between the determinant and partition function is the usual one based on the equivalence between path-integral formulation of quantum field theories and the operator formulation, when $\tau$ is purely imaginary. One simply views $2 \pi \tau_{2}$ as the time variable. In the case when $\tau$ also has a real part $\tau=\tau_{1}+i \tau_{2}$, the torus is slightly skewed and so the trace should be taken after multiplying by the operator which takes $\sigma_{1} \rightarrow \sigma_{1}+2 \pi \tau_{1}$. The operator which accomplishes this is $e^{2 \pi i \tau_{1}(H-\bar{H})}$, where $\bar{H}$ denotes the Hamiltonian for the other chirality. So altogether, we get $e^{2 \pi i \tau_{1}(H-\bar{H})-2 \pi \tau_{2}(H+\bar{H})}=q^{H} \bar{q}^{\bar{H}}$. Keeping one of the chiralities, we obtain the above result (multiplied by the $g$ twist operator).

In the above example, rather than changing the boundary conditions, we could fix the boundary conditions and instead introduce flat gauge fields which couple to the fermions. If we take the gauge field $A=\theta d \sigma_{1}-\phi d \sigma_{2}$, the chiral Dirac operator depends on the twistings only through the combination $\phi+\tau \theta$. So, defining the complex quantity $u$ by $u=\phi+\tau \theta$, we naively expect the determinant to be a function only of $u$ and not of its complex conjugate $\bar{u}$. In fact the explicit expression (2.1) for the $\operatorname{Det}(\theta, \phi)$ is almost of that form. The terms in the infinite product are only functions of $u$, but the quadratic term in the exponent of the $q$ term in front prevents us from writing the determinant solely as a function of $u$ :

$$
\theta^{2}=\left(\frac{1}{2 i \operatorname{Im} \tau}(u-\bar{u})\right)^{2}
$$

So it seems that there is some sort of anomaly. In fact this is an example of a holomorphic anomaly which will be discussed in detail in Sect. 4. 
It is possible to write the $(\theta, \phi)$ dependence of $\operatorname{Det}(\theta, \phi)$ in a way which generalizes to higher genus. For this purpose consider the function:

$$
\vartheta\left[\begin{array}{l}
\theta \\
\phi
\end{array}\right](0 \mid \tau)=\sum_{n} e^{i \pi(n+\theta)^{2} \tau+2 i \pi(n+\theta) \phi}=\sum_{n} q^{\frac{(n+\theta)^{2}}{2}} e^{2 \pi i(n+\theta) \phi} .
$$

Using the product representation of theta functions (2.1) becomes

$$
\operatorname{Det}(\theta, \phi)=\frac{\vartheta\left[\begin{array}{l}
\theta \\
\phi
\end{array}\right](0 \mid \tau)}{\eta(\tau)},
$$

where $\eta(\tau)$, is the Dedekind eta function:

$$
\eta(\tau)=q^{\frac{1}{24}} \prod_{n}\left(1-q^{n}\right)
$$

Therefore the twist dependence of the determinant is given by a theta function. Theta functions arise naturally in string theories as sums over winding sectors and internal momenta. The same is true here, and the right-hand side of (2.3) is in fact the twisted partition function of a single boson allowed to have momenta on a shifted lattice. The above identity is a statement about bosonization. To see this, note that the chiral fermionic current is replaced by the translation operator in the bosonized theory. The twist operator $g$ turns out to be given by $g=e^{2 \pi i \phi P}$, where $P$ is the translation operator, and we should require the bosonic momenta to lie on the shifted lattice $(n+\theta)$, where $n$ is any integer. The partition function $\operatorname{Tr} g q^{H}$, where $H=\frac{P^{2}}{2}+\sum n a_{n}^{\dagger} a_{n}$ with obvious commutation relations, is easily seen to be the expression (2.3). We will explore the analog of this argument for the higher genus case in Sect. 6.

In Sect. 7 we will discuss the transformation properties of theta functions and the modular invariance of multiloop string amplitudes. These matters are easily understood for the torus. It is straightforward to check, for example, that under modular transformations, $\vartheta\left[\begin{array}{l}0 \\ 0\end{array}\right], \vartheta\left[\begin{array}{l}0 \\ \frac{1}{2}\end{array}\right]$, and $\vartheta\left[\begin{array}{c}\frac{1}{2} \\ 0\end{array}\right]$ mix with each other, corresponding to the fact that all the even spin structures mix under global diffeomorphisms. $\vartheta\left[\begin{array}{c}\frac{1}{2} \\ \frac{1}{2}\end{array}\right]$ is zero, corresponding to the fact that the Dirac operator has zero modes. (If we delete the zero mode, the odd theta function is seen to transform to itself under modular transformations.)

The one-loop vacuum to vacuum amplitudes for superstrings are well-known. The contribution of the right-moving spinors is proportional to:

$$
\vartheta^{4}\left[\begin{array}{l}
0 \\
0
\end{array}\right]-\vartheta^{4}\left[\begin{array}{c}
\frac{1}{2} \\
0
\end{array}\right]-\vartheta^{4}\left[\begin{array}{c}
0 \\
\frac{1}{2}
\end{array}\right]
$$

We have five complex spin $\frac{1}{2}$ fermions and one complex spin $\frac{3}{2}$ ghost, which cancels the determinant of one of the fermions, to leave us with the power 4 for the theta function. This expression is modular covariant, i.e., under modular transformations it transforms to itself, up to a prefactor. In fact the above combination is 
equal to zero, and it implies that the cosmological constant vanishes, at one-loop order.

This concludes our discussion of fermions on a torus. We will see that many of these concepts are easily generalized to higher loops.

\section{Mathematical Background}

In this section we summarize some aspects of the theory of Riemann surfaces which are useful in the computation of higher loop string amplitudes. Since we only consider closed strings we will be concerned with compact Riemann surfaces. We will consider the topology, differential geometry, line bundles, and theta functions associated with a surface.

\section{a) The Mapping Class Group}

Topologically, orientable two-dimensional surfaces $\Sigma$ are completely classified by the Euler number $\chi(\Sigma)=2-2 g$, where $g$, the genus of $\Sigma$, is the number of handles (see Fig. 1). We describe first the homology of $\Sigma$.

When $\Sigma$ is compact the homology groups are free groups with dimensions

$$
\operatorname{dim} H_{0}(\Sigma)=1, \quad \operatorname{dim} H_{1}(\Sigma)=2 g, \quad \operatorname{dim} H_{2}(\Sigma)=1 .
$$

We can identify a canonical homology basis $a_{i}, b_{i}, 1 \leqq i \leqq g$ for $H_{1}(\Sigma)$ as in Fig. 1 . Then any closed curve on $\Sigma$ generates a homology class which can be uniquely decomposed in terms of the classes generated by $a_{i}, b_{i}$. The reason for calling $a_{i}, b_{i}$ a canonical basis is the following. If we define the intersection number $J\left(\gamma, \gamma^{\prime}\right)$ between two curves $\gamma$ and $\gamma^{\prime}$, as the number of points at which they intersect counting orientation, then, since the number $J\left(\gamma, \gamma^{\prime}\right)$ only depends on the homology classes generated by $\gamma$ and $\gamma^{\prime}, J$ defines a quadratic form on $H_{1}(\Sigma)$. In terms of the $a_{i}, b_{i}$ cycles, $J$ takes the canonical form

$$
J\left(a_{i}, a_{j}\right)=J\left(b_{i}, b_{j}\right)=0, \quad J\left(a_{i}, b_{j}\right)=-J\left(b_{i}, a_{j}\right)=\delta_{i j},
$$

or, as a matrix,

$$
J=\left(\begin{array}{rr}
0 & 1 \\
-1 & 0
\end{array}\right)
$$

Once we have chosen a canonical homology basis, we can represent $\Sigma$ by a $4 g$-sided polygon with appropriate identifications on the boundary. To do this, choose a point on $\Sigma$, and cut the surface along $2 g$ curves homologous to the canonical basis (this is depicted in Fig. 2 for the case of $g=2$ ). If in Fig. 2 we glue together the sides $a_{i} a_{i}^{-1}$ and $b_{i} b_{i}^{-1}$ we get back the original surface. Thus each handle is represented by the symbol $a_{i} b_{i} a_{i}^{-1} b_{i}^{-1}$.

We will often use the basis dual to the canonical homology basis. In terms of differential forms, we may use the Hodge-De Rham theory to set up a one to one

Fig. 1

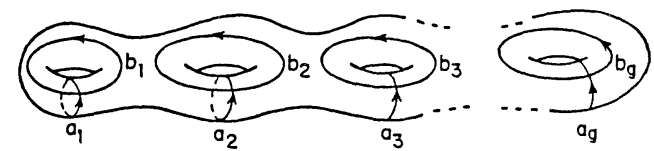


Fig. 2
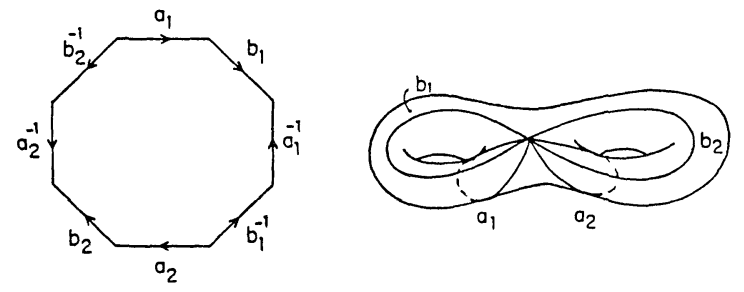

correspondence between elements in $H_{1}(\Sigma)$ and harmonic 1 -forms on $\Sigma$. Thus there are $2 g$ harmonic 1 -forms $\alpha_{i}, \beta_{i}, 1 \leqq i \leqq g$ normalized so that

$$
\begin{array}{ll}
\int_{a_{i}} \alpha_{j}=\delta_{i j}, & \int_{a_{i}} \beta_{j}=0, \\
\int_{b_{i}} \alpha_{j}=0, & \int_{b_{i}} \beta_{j}=\delta_{i j} .
\end{array}
$$

The representation of $\Sigma$ in terms of a polygon is very helpful in proving some useful identities. For example, if $\theta, \eta$ are closed 1 -forms then one can easily show [7]

$$
\int_{\Sigma} \theta \wedge \eta=\sum_{i=1}^{g}\left[\int_{a_{i}} \theta \int_{b_{i}} \eta-\int_{b_{i}} \theta \int_{a_{i}} \eta\right] .
$$

This completes our discussion of the homology and cohomology of $\Sigma$.

When we discuss global anomalies on the string world sheet and modular invariance of the string path integral we will need some facts about the nontrivial diffeomorphisms of $\Sigma$ which we now outline [8]. Even though the proofs of the statements which follow are nontrivial, the results themselves are easily described. Let $\operatorname{Diff}(\Sigma)$ be the group of diffeomorphisms of $\Sigma$, and let Diff ${ }_{0}(\Sigma)$ be the normal subgroup of diffeomorphisms homotopic to the identity. Then the mapping class group is defined by

$$
\Omega(\Sigma)=\operatorname{Diff}(\Sigma) / \operatorname{Diff}_{0}(\Sigma)
$$

We first describe the generators of $\Omega(\Sigma)$. These can be taken to be Dehn twists around closed curves $\gamma$. In general, a Dehn twist is defined by excising a small tubular neighborhood of $\gamma$, twisting one boundary of the tube by $2 \pi$, and glueing the tube back into the surface. This sequence of operations defines an active transformation of the surface to itself (see Fig. 3). A useful result is that a set of

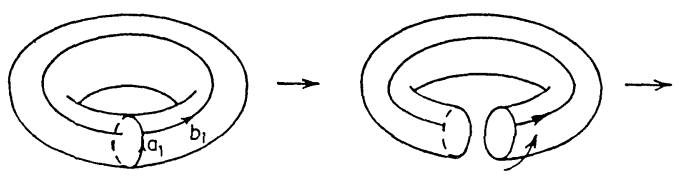

Fig. 3

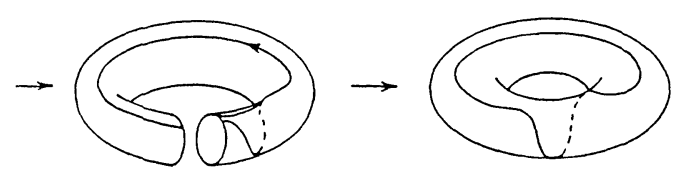


Fig. 4

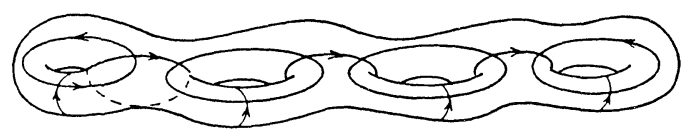

generators of $\Omega(\Sigma)$ is provided by the Dehn twists about the curves illustrated in Fig. 4. (This is not, in fact, the minimal set.) Thus we have two generators for each handle (as on the torus) and a generator for the curve linking the holes of two consecutive handles. Thus, using the labeling of the homology basis in Fig. 1, the generators of $\Omega(\Sigma)$ are twists around

$$
a_{1}, b_{1}, a_{1}^{-1} a_{2}, a_{2}, b_{2}, a_{2}^{-1} a_{3}, \ldots, a_{g}, b_{g} .
$$

Thus it suffices to check modular invariance of the string integrand by checking modular invariance under these diffeomorphisms. We will apply this remark to obtain nontrivial restrictions on heterotic string theories in Sect. 7.

A useful representation of nontrivial diffeomorphisms is provided by their action on the homology of $\Sigma$. If the curve $\gamma$ generates a nontrivial homology class, then a Dehn twist around $\gamma$ acts nontrivially on the homology basis. For instance, in Fig. 3 the Dehn twist around $a_{1}$ induces the following transformation on the homology: $a_{1} \rightarrow a_{1}, b_{1} \rightarrow b_{1}+a_{1}$. Let $D_{\gamma}$ be the diffeomorphism defined by the twist around $\gamma$. The intersection matrix is manifestly invariant under diffeomorphisms, so the action of $\Omega(\Sigma)$ on the homology group $H_{1}(\Sigma, Z)$ must necessarily preserve (3.2). Thus, the matrix $M\left(D_{\gamma}\right)$ representing the action of $D_{\gamma}$ on $H_{1}(\Sigma, Z)$ is a nonsingular $2 g \times 2 g$ matrix with integer entries, leaving the symplectic form (3.2) invariant, i.e. $M\left(D_{\gamma}\right)$ is an element of $S p(2 g, Z)$, the group of integer symplectic matrices, also known as the symplectic modular group. An important result is that the set of matrices $M\left(D_{\gamma}\right)$ in fact generate all of $\operatorname{Sp}(2 g, Z)$ [9].

As an example we construct an explicit representation of the generators of $\Omega(\Sigma)$ for $g=2$. They are given by the following $4 \times 4$ matrices:

$$
\begin{aligned}
& D_{a_{1}}=\left(\begin{array}{cccc}
1 & 0 & 0 & 0 \\
0 & 1 & 0 & 0 \\
1 & 0 & 1 & 0 \\
0 & 0 & 0 & 1
\end{array}\right), \quad D_{b_{1}}=\left(\begin{array}{cccc}
1 & 0 & 1 & 0 \\
0 & 1 & 0 & 0 \\
0 & 0 & 1 & 0 \\
0 & 0 & 0 & 1
\end{array}\right), \\
& D_{a_{2}}=\left(\begin{array}{cccc}
1 & 0 & 0 & 0 \\
0 & 1 & 0 & 0 \\
0 & 0 & 1 & 0 \\
0 & 1 & 0 & 1
\end{array}\right), \quad D_{b_{2}}=\left(\begin{array}{cccc}
1 & 0 & 0 & 0 \\
0 & 1 & 0 & 1 \\
0 & 0 & 1 & 0 \\
0 & 0 & 0 & 1
\end{array}\right) \text {, } \\
& D_{a_{1}{ }_{1}^{1} a_{2}}=\left(\begin{array}{rrrr}
1 & 0 & 0 & 0 \\
0 & 1 & 0 & 0 \\
-1 & 1 & 1 & 0 \\
1 & -1 & 0 & 1
\end{array}\right)
\end{aligned}
$$

An explicit construction of the action of $D_{a_{1}^{-1} a_{2}}$ on the homology is illustrated in Fig. 5. 

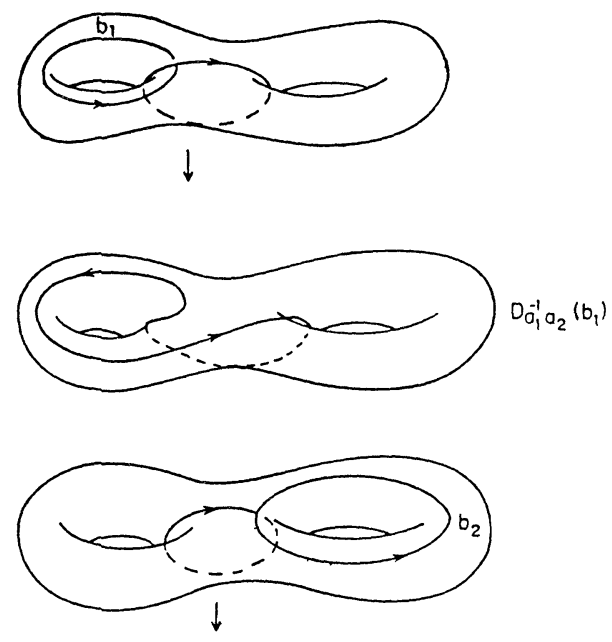

Fig. 5

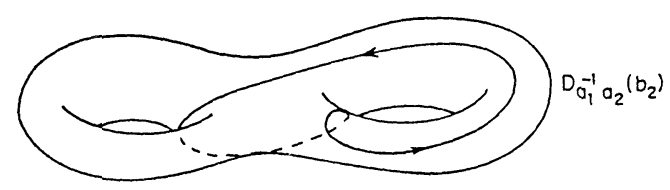

\section{b) Differential Geometry and Spin Structures}

In this section we will define spin structures and review some of the elementary differential geometry of Riemann surfaces. Thus far we have considered $\Sigma$ as a topological surface. The surfaces relevant to string theory are also endowed with a complex structure, which always exists if $\Sigma$ is orientable. We will view the complex structure of $\Sigma$ as that defined by a Riemannian metric $g$. Locally, we can always write any metric on $\Sigma$ in the form

$$
d s^{2}=e^{2 \phi}\left[\left(d x^{0}\right)^{2}+\left(d x^{1}\right)^{2}\right]=2 g_{w \bar{w}} d w d \bar{w}
$$

with $w=x^{0}+i x^{1}, \bar{w}=x^{0}-i x^{1}$. When we cover $\Sigma$ with such complex coordinate patches $\mathscr{U}$, the transition functions on the overlaps are holomorphic, and thus define a complex structure. Using this complex structure we can divide the 1 -forms

$$
T^{*} \Sigma=T^{*(1,0)} \Sigma \oplus T^{*(0,1)} \Sigma
$$

according to whether they are locally of the form $f(w, \bar{w}) d w$ or $f(w, \bar{w}) d \bar{w}$. In particular, there are $g(1,0)$ - forms $\omega_{i}$ which are holomorphic, i.e., are locally of the form $f_{i}(w) d w$ where $f_{i}$ is holomorphic. A convenient way of normalizing the $\omega_{i}$ (also known as abelian differentials of the first kind) is to require

$$
\int_{a_{i}} \omega_{j}=\delta_{i j}
$$

This completely specifies the $\omega_{i}[7]$. One then finds for the $b_{i}$ cycles

$$
\int_{b_{i}} \omega_{j}=\Omega_{i j} \text {. }
$$


Using (3.4) with $\theta=\omega_{i}, \eta=\omega_{j}$, it follows that $\Omega_{i j}=\Omega_{j i}$, and if one uses again (3.4) with $\theta=\bar{\eta}$, and $\eta$ holomorphic, then

$$
\frac{1}{2 i} \int \bar{\theta} \wedge \theta=\operatorname{Im} \sum_{i} \overline{\int_{a_{i}} \theta} \int_{b_{i}} \theta>0,
$$

and we see that $\operatorname{Im} \Omega>0$. The matrix $\Omega$ is thus a $g \times g$ complex symmetric matrix with positive imaginary part, known as the period matrix of the Riemann surface. The space of all matrices satisfying these conditions is known as the Siegel upper half plane $\mathscr{H}_{g}$ (in analogy with the $g=1$ case where $\Omega$ is the standard modular parameter of the torus).

Tensor algebra and analysis are particularly simple on a two-dimensional surface. By raising and lowering indices with the metric (3.5) any tensor can be decomposed into higher order differentials $\psi(d w)^{n}, n \in Z$. The transformation properties of $\psi$ in going from a patch with complex coordinate $w$ to another with coordinate $u$ are obtained by requiring

$$
\psi^{\prime}(w)(d w)^{n}=\psi(u)(d u)^{n} \Rightarrow \psi^{\prime}(w)=\left(\frac{d u}{d w}\right)^{n} \psi(u) .
$$

In order to introduce spinors we choose local frames

$$
d s^{2}=\delta_{a b} e^{a} \otimes e^{b} .
$$

If $\mathscr{U}_{\alpha}, \mathscr{U}_{\beta}$ are two overlapping coordinate patches, the frames $e_{(\alpha)}, e_{(\beta)}$ are related by a local $S O(2)$ rotation:

$$
e_{(\alpha)}^{a}=R^{a b}(\theta) e_{(\beta)}^{b},
$$

then the spinor bundles have transition functions $\tilde{R}(\theta / 2)$, so that $(\tilde{R}(\theta / 2))^{2}=R(\theta)$. If we consider three overlapping patches $\mathscr{U}_{\alpha}, \mathscr{U}_{\beta}, \mathscr{U}_{\gamma}$ the cocycle condition $R_{\alpha \beta} R_{\beta \gamma} R_{\gamma \alpha}=1$, imposes a nontrivial constraint on $\widetilde{R}$, namely,

$$
\tilde{R}_{\alpha \beta} \widetilde{R}_{\beta \gamma} \tilde{R}_{\gamma \alpha}=w_{2}(\alpha, \beta, \gamma)
$$

is a two-cocyle with $Z_{2}$ coefficients (i.e. for any three overlapping patches $\alpha, \beta, \gamma$, $\left.w_{2}= \pm 1\right), w_{2}$ is the second Stieffel-Whitney class. If $w_{2}$ is not cohomologous to zero, then the surface does not admit a spin structure. Since $w_{2}$ is the reduction modulo two of the Euler character [10], which is even for a compact Riemann surface, we know that $w_{2}$ is a coboundary: $w_{2}=\delta \eta$. Hence spinor structures always exist. The number of inequivalent spin structures is then the number of solutions to the equation $\delta\left(\eta-\eta^{\prime}\right)=0$, i.e., the number of $Z_{2}-1$-cocycles. These span the space $H^{1}\left(\Sigma, Z_{2}\right)$. Thus, even though describing a spin structure explicitly may in general be awkward, the difference between two spin structures is simply a question of assigning plus or minus signs to the generators of the homology of $\Sigma$. This implies in particular that $\Sigma$ has $2^{2 g}$ inequivalent spin structures.

One of the pleasant features of Riemann surfaces is that we can describe spinors in terms of half-order differentials. This is most easily done by choosing frames:

$$
\begin{aligned}
& e^{z}=e^{0}+i e^{1}=e^{\phi} d w \quad \text { for } \quad T^{*(1,0)}, \\
& e^{\bar{z}}=e^{0}-i e^{1}=e^{\phi} d \bar{w} \quad \text { for } \quad T^{*(0,1)},
\end{aligned}
$$


where $\phi$ is the factor in (3.5). Then across patches $\mathscr{U}_{\alpha}, \mathscr{U}_{\beta}$ with coordinates $w_{\alpha}, w_{\beta}$

$$
e^{2 \phi_{\alpha}}\left|d w_{\alpha}\right|^{2}=e^{2 \phi_{\beta}}\left|d w_{\beta}\right|^{2} \text {, }
$$

which implies

$$
2 \phi_{\alpha}=2 \phi_{\beta}+\log \left|\frac{d w_{\beta}}{d w_{\alpha}}\right|^{2}
$$

so we have

$$
e_{(\alpha)}^{z}=e^{i \theta} e_{(\beta)}^{z}, \quad e^{i \theta}=\frac{d w_{\alpha}}{d w_{\beta}}\left|\frac{d w_{\beta}}{d w_{\alpha}}\right|,
$$

and the left and right spinors $\psi \in S^{ \pm}$transform as

$$
\psi_{ \pm \alpha}=e^{ \pm i \theta / 2} \eta_{\alpha \beta} \psi_{ \pm \beta} .
$$

Here again the cocycle $\eta$ gives the spin structure relative to one particular choice of square roots of $e^{i \theta}$. When we refer the spinors $\psi_{ \pm}$to the frames $\left(e^{z}\right)^{1 / 2},\left(e^{\bar{z}}\right)^{1 / 2}$ the transition functions are one-by-one unitary matrices. It is occasionally more convenient to consider the bundles $S^{ \pm}$as holomorphic line bundles. These will have transition functions $\eta_{\alpha \beta}\left(d w_{\alpha} / d w_{\beta}\right)^{1 / 2}$ for $S^{+}$and $\eta_{\alpha \beta}\left(d w_{\alpha} / d w_{\beta}\right)^{-1 / 2}$ for $S^{-}$. In the holomorphic category more appropriate local sections are the holomorphic halforder differentials $\left(d w_{\alpha}\right)^{1 / 2}$. The relation between the standard and holomorphic descriptions of spinors is given by

$$
\psi_{+}\left(e^{z}\right)^{1 / 2}=\psi_{\theta}(d w)^{1 / 2} .
$$

The holomorphic line bundle defined by $S^{+}$will be denoted by $L$. As suggested by the notation, this bundle can be interpreted as a holomorphic square root of the bundle of $(1,0)$-forms:

$$
T^{*(1,0)} \Sigma=K=L_{\alpha}^{2},
$$

here $\alpha$ labels the spin structure. Since there are $2^{2 g}$ spin structures $K$ will admit $2^{2 g}$ inequivalent holomorphic square roots.

Once we have introduced the spinor bundle $L_{\alpha}$, we can define tensor powers $L_{\alpha}^{n}$ corresponding to differentials $\psi\left(d w_{\alpha}\right)^{n / 2}$. In the local coordinates (3.5) the covariant derivative for fields in $L_{\alpha}^{n}$ is:

$$
\nabla_{w}^{n}: L_{\alpha}^{n} \rightarrow L_{\alpha}^{n+2},
$$

where

$$
\nabla_{w}^{n} \psi=\left(g_{w \bar{w}}\right)^{n / 2} \frac{\partial}{\partial w}\left(g_{w \bar{w}}\right)^{-n / 2} \psi .
$$

We can introduce a scalar product in $L_{\alpha}^{n}$ :

$$
\langle\phi \mid \psi\rangle=\int d^{2} \sigma \sqrt{g}\left(g^{w \bar{w}}\right)^{n / 2} \phi^{*} \psi .
$$

The operator $\nabla_{w}^{n}$ is just the unique holomorphic connection on $L_{\alpha}^{n}$ compatible with the metric (3.11). With respect to (3.11) the adjoint of (3.10) is

$$
\begin{aligned}
& \nabla_{n+2}^{w}=\left(\nabla_{w}^{n}\right)^{\dagger}: L_{\alpha}^{n+2} \rightarrow L_{\alpha}^{n}, \\
& \nabla_{n+2}^{w}=-g^{w \bar{w}} \frac{\partial}{\partial \bar{w}} \psi .
\end{aligned}
$$


With (3.10) and (3.12) we can construct two Laplacians:

$$
\begin{aligned}
& \Delta_{n}^{(+)}=\nabla_{n+2}^{w} \nabla_{w}^{n}, \\
& \Delta_{n}^{(-)}=\nabla_{w}^{n-2} \nabla_{n}^{w} .
\end{aligned}
$$

It is easy to show that $\Delta_{n}^{(+)}$and $\Delta_{n+2}^{(-)}$have the same spectrum of non-vanishing eigenvalues. For the zero-modes, the Riemann-Roch theorem states

$$
\text { ind } \nabla_{w}^{n} \equiv \operatorname{dimker} \nabla_{w}^{n}-\operatorname{dimker} \nabla_{n+2}^{w}=-(n+1)(g-1) \text {. }
$$

When $g>1$ any Riemann surface admits a natural metric with constant negative curvature. Since

$$
\Delta_{n}^{(+)}=\Delta_{n}^{(-)}-\frac{n}{4} R
$$

where

$$
R=-2 e^{-2 \phi} \partial_{w} \partial_{\bar{w}} \phi,
$$

and since $\Delta_{n}^{( \pm)}$are both positive definite operators, we conclude that for $R<0$, and $n \geqq 1$, the dimension of $\operatorname{ker} \nabla_{u}^{n}$ is zero, and

$$
\operatorname{dim} \operatorname{ker} \nabla_{n+2}^{w}=(n+1)(g-1) \text {. }
$$

\section{c) Divisors and Line Bundles}

We now describe some aspects of the general theory of holomorphic line bundles on $\Sigma$. In the next section we will outline their relationship to Riemann's theta function. A holomorphic line bundle can be defined by its transition functions: $\psi_{\alpha}=g_{\alpha \beta} \psi_{\beta}$. (The transition functions $g_{\alpha \beta}$ are nowhere vanishing holomorphic functions on the overlaps $\mathscr{U}_{\alpha} \cap \mathscr{U}_{\beta}$.) There is an alternative description of $L$ in terms of so-called divisors. Given a meromorphic section, $\psi$, of $L$ we can consider the set of points where $\psi$ vanishes or blows up: $\left\{P_{i}\right\}$. Since $n_{i}$, the order of the zero or pole of $\psi$ at $P_{i}$ is independent of the trivialization of $L$, we can define the divisor of $\psi$ as the formal sum $\operatorname{div}(\psi)=\sum n_{i} P_{i}$. Another section of the same line bundle $L$ is obtained if we multiply $\psi$ by any meromorphic function on $\Sigma$ (recall that a meromorphic function $f$ has the same number of zeroes and poles, and that $f$ is determined up to a constant by its divisor). Given this ambiguity in the construction of a section, we define an equivalence relation between two divisors $D_{1}$ and $D_{2}$ by $D_{1} \sim D_{2}$ if $D_{1}-D_{2}$ is the divisor of a meromorphic function. Thus, to a given line bundle $L$, we can associate a divisor class $(L)$. Conversely, given a divisor class $(D)$, we can construct a line bundle $L$, as follows. Choose a representative divisor $D$ of the class $(D)$. On any patch $\mathscr{U}_{\alpha}$, we can find a meromorphic function $f_{\alpha}$ whose divisor on $\mathscr{U}_{\alpha}$ coincides with the restriction of $D$ to $\mathscr{U}_{\alpha}$. Then, on the overlap $\mathscr{U}_{\alpha} \cap \mathscr{U}_{\beta}, f_{\alpha}$ and $f_{\beta}$ represent the same divisor, so $g_{\alpha \beta}=f_{\alpha} / f_{\beta}$ is a nowhere vanishing holomorphic function, and it trivially satisfies the cocycle condition. Hence $D$ defines a line bundle $L$. This construction shows that there is a one-to-one correspondence between divisor classes and holomorphic line bundles on a Riemann surface. The degree of a divisor $D=\sum n_{i} P_{i}$ is defined as $\operatorname{deg} D=\sum n_{i}$. The degree only depends on the divisor class, and coincides with the first Chern 
class of $L$. For example, the first Chern class of the spinor bundle $L_{\alpha}$ can be found by noting that $L_{\alpha}^{2}=K$, and recalling that the first Chern class of $K$ is minus the Euler class. Hence $c_{1}\left(L_{\alpha}\right)=g-1$.

The concept of a divisor generalizes to higher dimensions. A divisor on a complex manifold is a formal sum $\sum n_{i} V_{i}$, where $V_{i}$ are complex codimension one analytic subvarieties. (A variety is a generalization of a manifold which allows certain singularities.) The equivalence between divisor classes and line bundles described above continues to hold in the higher dimensional case. Technical details can be found in standard texts [11]. This concludes our introduction to divisors.

Differentiable complex line bundles are completely classified by their first Chern class, but the space of holomorphic line bundles has more structure. We will now briefly outline the classification of holomorphic complex line bundles [12]. The difference $L_{1} \otimes L_{2}^{-1}$ of two line bundles on a Riemann surface with the same Chern class is a holomorphic line bundle which admits a flat connection. It can be shown that the group of flat line bundles is a torus, known as the Picard variety of $\Sigma$, and denoted by $\operatorname{Pic}(\Sigma)$. We can describe this space intuitively as follows. We cut $\Sigma$ along a basis $a_{i}, b_{i}$ for $H_{1}(\Sigma, Z)$ as in Fig. 2. The sections of a flat bundle are then characterized by their transition functions around $a_{i}$ and $b_{i}$, which can be taken to be constant phases. Thus we identify the section $\psi$ along $a_{i}$ with $e^{-2 \pi i \varphi_{i}}$ times $\psi$ along $a_{i}^{-1}$, and $\psi$ along $b_{i}$ with $e^{2 \pi i \theta_{i}}$ times $\psi$ along $b_{i}^{-1}$. In other words, flat holomorphic line bundles are completely classified by their twists on the homology, and these twists are parametrized by the torus $R^{2 g} / Z^{2 g}$. Therefore a holomorphic line bundle is completely characterized by an integer (its first Chern class) and a point on the Picard variety which determines the twists on the homology. For example, if we denote by $V(\boldsymbol{\theta}, \boldsymbol{\phi})$ the flat line bundle with twists $e^{2 \pi i \theta_{k}}, e^{-2 \pi i \varphi_{k}}$, then the line bundles of degree $1-g$ can be parametrized by $L_{\alpha}^{-1} \otimes V(\boldsymbol{\theta}, \phi)$, where $L_{\alpha}^{-1}$ is some fixed spin structure. From (3.9) we see that twice the difference of spin bundles is trivial. Thus, in this parametrization of degree $1-g$ line bundles the points corresponding to spin structures are the half-points of the torus, that is, the points where $(\theta, \phi) \in\left(\frac{1}{2} Z / Z\right)^{2 g}$.

In Sect. 5 we will compute the spin-structure dependence of the determinant of the Dirac operator. One important ingredient will be a trick which we now discuss. Recall that the chiral Dirac operator is the holomorphic connection on $L_{\alpha}^{-1}$ compatible with the metric (3.11). Therefore, we consider the holomorphic connections on $L_{\alpha}^{-1} \otimes V(\boldsymbol{\theta}, \boldsymbol{\phi})$. Again considering $\Sigma$ cut along its homology basis, we see that eigenfunctions of $\not D$ in $L_{\alpha}^{-1} \otimes V(\theta, \phi)$ are related by the unitary transformation

$$
\mathscr{U}(P)=\exp \left(\int_{P_{0}}^{P} A\right)
$$

to eigenfunctions of the coupled Dirac operator $\not_{A}$ in $L_{\alpha}^{-1}$, where the gauge field $A$ can be constructed in terms of the harmonic one-forms on $\Sigma$ :

$$
A=2 \pi i \sum_{1}^{g} \theta^{i} \alpha_{i}-2 \pi i \sum_{1}^{g} \phi^{i} \beta_{i} .
$$


Locally, $A(\boldsymbol{\theta}, \boldsymbol{\phi})$ is pure gauge, but it cannot be gauged away because it has nontrivial holonomy. The advantage of introducing the gauge field (3.13) is that if we compute $\operatorname{det} \not_{A}$ as a function of $A$ for a given spin structure, then evaluating the answer for the points $(\boldsymbol{\theta}, \boldsymbol{\phi})$ in $\left(\frac{1}{2} Z / Z\right)^{2 g}$ we have effectively computed the spinstructure dependence of $\operatorname{det} \not D$. Thus there are two equivalent procedures for computing the determinant of the Dirac operator on all line bundles of degree $1-g$. The first is to keep the operator $\nabla_{w}^{-1}$ "fixed" and vary the line bundle $L_{\alpha}^{-1} \otimes V(\theta, \phi)$, and the second is to keep the line bundle $L_{\alpha}^{-1}$ fixed and vary the operator by including the flat $U(1)$ gauge field $A$. We will usually adopt the second point of view.

We can write $A$ in a more useful way in terms of the abelian differentials. It is easy to show that the $\alpha_{i}, \beta_{i}$ and the $\omega_{i}, \bar{\omega}_{i}$ are related by

$$
\begin{aligned}
& \alpha_{i}=A_{i j} \omega_{j}+\text { cplx. conj. } \\
& \beta_{i}=B_{i j} \omega_{j}+\text { cplx. conj. }
\end{aligned}
$$

where

$$
A=-\bar{\Omega}(\Omega-\bar{\Omega})^{-1}, \quad B=(\Omega-\bar{\Omega})^{-1},
$$

so that

$$
A=2 \pi i(\boldsymbol{\phi}+\Omega \boldsymbol{\theta}) \cdot(\Omega-\bar{\Omega})^{-1} \cdot \bar{\omega}+\text { h.c. } .
$$

Thus the chiral Dirac operator $\nabla_{w}^{-1}: L_{\alpha}^{-1} \rightarrow L_{\alpha}$ when coupled to $A$ becomes

$$
D(\mathbf{u})=\nabla_{w}^{-1}-2 \pi i(\boldsymbol{\phi}+\bar{\Omega} \boldsymbol{\theta}) \cdot(\Omega-\bar{\Omega})^{-1} \cdot \omega,
$$

where $\nabla_{w}^{-1}$ is the operator introduced in (3.10). Thus we have a family of Dirac operators parametrized by $\mathbf{u}=\boldsymbol{\phi}+\overline{\boldsymbol{\Omega}} \boldsymbol{\theta}$. Since $\boldsymbol{\phi} \rightarrow \boldsymbol{\phi}+\mathbf{m}, \boldsymbol{\theta} \rightarrow \boldsymbol{\theta}+\mathbf{n}$ for $\mathbf{n}, \mathbf{m}$ integral vectors defines the same point on $\operatorname{Pic}(\Sigma)$, the family is parametrized by another complex torus known as the Jacobian variety of $\Sigma$, denoted by $J(\Sigma)$, and defined by $J(\Sigma)=C^{g} / L_{\Omega}$, where $L_{\Omega}$ is the lattice generated by $Z^{g}+\Omega Z^{g}$. Since $\Omega$ (the period matrix) is a non-singular $g \times g$ complex matrix, there is a $1-1$ correspondence between $\operatorname{Pic}(\Sigma)$ and $J(\Sigma)$.

\section{d) Theta Functions}

In this section we define the theta functions and explain their relation to spin structures. We follow the notation and ireatment of Mumford [13]. Another excellent reference is the book of Fay [14]. In analogy with the genus one case, once we have introduced the Jacobian $J(\Sigma)$, we can introduce the Riemann theta function by the series expansion:

$$
\vartheta(\mathbf{z} \mid \Omega)=\sum_{\mathbf{n} \in \mathbb{Z}^{g}} \exp (i \pi \mathbf{n} \cdot \Omega \cdot \mathbf{n}+2 \pi i \mathbf{n} \cdot \mathbf{z}) .
$$

We also define theta functions with characteristics $\mathbf{a}, \mathbf{b}$ for $\mathbf{a}, \mathbf{b} \in R^{g}$ by the sum

$$
\begin{aligned}
\vartheta\left[\begin{array}{l}
\mathbf{a} \\
\mathbf{b}
\end{array}\right](\mathbf{z} \mid \Omega) & =\sum_{\mathbf{n} \in \mathcal{Z}^{g}} \exp (i \pi(\mathbf{n}+\mathbf{a}) \cdot \Omega \cdot(\mathbf{n}+\mathbf{a})+2 \pi i(\mathbf{n}+\mathbf{a}) \cdot(\mathbf{z}+\mathbf{b})) \\
& =e^{i \pi \cdot \Omega \cdot \Omega \cdot a+2 \pi i a \cdot(z+b)} \vartheta(\mathbf{z}+\Omega \mathbf{a}+\mathbf{b} \mid \Omega)
\end{aligned}
$$


if we shift $\mathbf{z}$ by the lattice $L_{\Omega}, \vartheta$ transforms by

$$
\vartheta\left[\begin{array}{l}
\mathbf{a} \\
\mathbf{b}
\end{array}\right](\mathbf{z}+\Omega \mathbf{n}+\mathbf{m} \mid \Omega)=e^{-i \pi n \cdot \Omega \cdot n-2 \pi i n \cdot(z+b)} e^{2 \pi i a \cdot m} \vartheta\left[\begin{array}{l}
\mathbf{a} \\
\mathbf{b}
\end{array}\right](\mathbf{z} \mid \Omega) .
$$

Another useful transformation law is:

$$
\vartheta\left[\begin{array}{c}
\mathbf{a}+\mathbf{n} \\
\mathbf{b}+\mathbf{m}
\end{array}\right](\mathbf{z} \mid \Omega)=e^{2 \pi i a \cdot m} \vartheta\left[\begin{array}{l}
\mathbf{a} \\
\mathbf{b}
\end{array}\right](\mathbf{z} \mid \Omega) .
$$

Thus the theta function is not strictly a function on $J(\Sigma)$; rather, it is a section of a holomorphic line bundle on $J(\Sigma)$ called the $\vartheta$-line bundle, $\mathscr{L}$. From the transition functions (3.18) one can compute the first Chern class of $\mathscr{L}$. Alternatively we can define the hermitian norm on sections of $\mathscr{L}$ by

$$
\|S\|^{2}=e^{i \pi(u-\bar{u}) \cdot(\Omega-\bar{\Omega})^{-1} \cdot(u-\bar{u})}|S|^{2}
$$

and compute the curvature

$$
c_{1}(\mathscr{L})=\frac{i}{2 \pi} \partial \bar{\partial} \log \|s\|^{2}=\sum_{1}^{g} d \phi^{i} \wedge d \theta^{i} .
$$

We can now apply the Kodaira vanishing theorem [11] and the index theorem for the $\bar{\partial}$-complex coupled to $\mathscr{L}$ to show that $\mathscr{L}$ admits only one holomorphic section which is represented by the theta function. ${ }^{1}$

The relation between spinor bundles and theta functions is based on Riemann's vanishing theorem. To state this fundamental theorem we first introduce the Jacobian map of $\Sigma$ into $J(\Sigma)$. Given a canonical homology basis, and a point $P_{0}$ on $\Sigma$, we can associate to any point $P$ on $\Sigma$ a point in $J(\Sigma)$ by

$$
\Phi_{i}(P)=\int_{P_{0}}^{P} \omega_{i},
$$

where the $\omega_{i}$ are the holomorphic differentials. As a map from $\Sigma$ into $C^{g}, \Phi$ is multivalued, but, considered as a map into $J(\Sigma)$ it is single-valued since if we move $P$ around one of the cycles $a_{i}$ or $b_{i}$, then (3.22) changes by an element of the Jacobian lattice. Similarly, the function on $\Sigma$ defined by

$$
f(P)=\vartheta\left(\mathbf{z}+\int_{P_{0}}^{P} \omega \mid \Omega\right)
$$

for $\mathbf{z}$ in $C^{g}$ is multivalued. However, $f$ is a well-defined function on the interior of the region in Fig. 2. By repeatedly applying Green's theorem to the one-form $d f / f$ on this region one can prove the

Riemann Vanishing Theorem. The function $f(P)$ either vanishes identically for all $P \in \Sigma$ or $f(P)$ has exactly g zeroes $P_{1}, \ldots, P_{g}$ on $\Sigma$. Furthermore, in the latter case, there exists a vector $\Delta$ depending only on $P_{0}$ and the canonical homology basis so that the points $\left\{P_{i}\right\}$ satisfy

$$
\mathbf{z}+\sum_{i=1}^{g} \int_{P_{0}}^{P_{i}} \omega=\Delta
$$

\footnotetext{
${ }^{1}$ We thank Dan Freed for explaining this to us
} 
Conversely, for all $P_{1}, \ldots, P_{g} \in \Sigma$, if we define $\mathbf{z}$ according to (3.24) then $f\left(P_{i}\right)=0$ for all $i=1, \ldots, g$.

$\Delta$ is known as the vector of Riemann constants. Since we will not need the explicit form of $\Delta$ we refer to $[13,14]$ for details, and the proof of this result.

The set of points $\mathbf{z} \in J(\Sigma)$, where $\vartheta(\mathbf{z} \mid \Omega)=0$, known as the $\Theta$-divisor, is a variety of complex codimension one. $\Theta$ will be important to us in Sect. 5. The Riemann vanishing theorem leads to the following description of $\Theta$ :

Corollary. $\vartheta(\mathbf{e} \mid \Omega)=0$ iff there exist $g-1$ points $P_{1}, \ldots, P_{g-1} \in \Sigma$ so that

$$
\mathbf{e}=\Delta-\sum_{1}^{g-1} \int_{P_{0}}^{P_{i}} \boldsymbol{\omega} .
$$

One direction of the proof of the corollary is simple. If $\mathbf{e}$ is given by (3.25) then we choose any point $P_{g} \in \Sigma$ and set

$$
\mathbf{z}=\Delta-\sum_{1}^{g} \int_{P_{0}}^{P_{i}} \omega .
$$

Then the Riemann vanishing theorem implies:

$$
0=f\left(P_{g}\right)=\vartheta\left(\mathbf{z}+\int_{P_{0}}^{P_{g}} \mathbf{\omega} \mid \Omega\right)=\vartheta(\mathbf{e} \mid \Omega) .
$$

With a little more work one can show the converse [13].

The sums which arise in the above theorems are clearly related to divisors. In particular, the divisor $\sum_{1}^{g-1} P_{i}$ defines a (possibly twisted) spin bundle. Thus the connection with spinors begins to emerge. The relation can be made more precise by generalizing the Jacobian map (3.22) to a map $I$ from divisors of degree zero to $J(\Sigma)$ as follows. If $D$ is a degree zero divisor, we choose a 1-cycle $\sigma$ on $\Sigma$ whose boundary is $D$, i.e. $\partial \sigma=D$. Then the Jacobian map is just

$$
I(D)=\int_{\sigma} \boldsymbol{\omega} \in J(\Sigma) .
$$

Note that the choice of cycle does not matter in (3.26). Two important properties of $I$ are,

i) Abel's Theorem. $I(D)=0$ if and only if $D$ is the divisor of a meromorphic function. Thus I is defined on divisor classes.

ii) Jacobi Inversion Theorem. The set of points $I\left[\sum_{1}^{g} P_{i}-g P_{0}\right]$ for $P_{1}, \ldots, P_{g} \in \Sigma$ is all of $J(\Sigma)$.

We are finally in a position to relate spin structures to $\Theta$. We begin with the remark that, by the index theorem, a degree $g-1$ bundle $E$ (that is, a twisted spin bundle) will have a holomorphic section if and only if the bundle $K^{-1} \otimes E$ has a zero-mode of $\nabla_{w}$. We can take the complex conjugate of the zero-mode equation [considering $K^{-1} \otimes E$ as a $U(1)$ bundle] and translate back into holomorphic language to see that $E$ has a holomorphic section if and only if $K \otimes E^{-1}$ has a holomorphic section. That is, $E$ has a divisor of the form $P_{1}+\ldots P_{g-1}$ if and only if $K \otimes E^{-1}$ has a divisor of the form $Q_{1}+\ldots Q_{g-1}$. Since a spin structure $L_{\alpha}$ satisfies 
$L_{\alpha}^{2}=K$, one can show that the set of points

$$
S_{\alpha}=\left\{I\left[P_{1}+\ldots P_{g-1}-D_{\alpha}\right] \mid P_{1}, \ldots, P_{g-1} \in \Sigma\right\},
$$

where $D_{\alpha}$ is a divisor of $L_{\alpha}$, is a symmetric subset of $J(\Sigma)$. However, from the Riemann vanishing theorem we see that the set $S_{\alpha}$ is just a translate of $\Theta$ by $\Delta-I\left[D_{\alpha}-(g-1) P_{0}\right]$. Thus, to any spin structure we may associate a symmetric translate of $\Theta$. That is, if $\mathbf{e}$ is in $S_{\alpha}$, then so is - e.

On the other hand, since $\vartheta$ is an even function of $\mathbf{z}, \Theta$ is itself symmetric. Therefore, a translate $\Theta+\mathbf{e}$ of $\Theta$ is symmetric if $\Theta+2 \mathbf{e}=\Theta$. An application of Liouville's theorem to the function

$$
\frac{\vartheta(\mathbf{z}+2 \mathbf{e})}{\vartheta(\mathbf{z})}
$$

on $C^{g}$ shows that $2 \mathbf{e} \in L_{\Omega}$. Thus the symmetric translates of $\Theta$ are the translates by points of order two.

Finally, recall that the difference of two spin structures $D_{1}-D_{2}$ is a point of order two in the Picard variety, hence $I\left[D_{1}-D_{2}\right]$ is of order two in $J(\Sigma)$. Thus each of the $2^{2 g}$ spin structures corresponds to one of the $2^{2 g}$ half-points in $J(\Sigma)$. In particular, some spin structure must correspond to the symmetric translate of $\Theta$ given by $\Theta$ itself. We call this spin structure $D_{0}$, i.e.

$$
\Delta=I\left[D_{0}-(g-1) P_{0}\right]
$$

The vector of Riemann constants depends on $P_{0}$, but $D_{0}$ does not. Both $\Delta$ and $D_{0}$ depend on the choice of canonical homology basis. ${ }^{2}$

The correspondences we have just outlined allow a division of spin structures into two classes known as even and odd spin structures. Note that if $\left(\boldsymbol{\varepsilon}_{1}, \boldsymbol{\varepsilon}_{2}\right) \in\left(\frac{1}{2} Z / Z\right)^{2 g}$ is a half-point then the divisor of $\vartheta\left[\begin{array}{l}\varepsilon_{1} \\ \varepsilon_{2}\end{array}\right](\mathbf{z} \mid \Omega)$ is a symmetric translate of $\Theta$. Also, it is straightforward to show that

$$
\vartheta\left[\begin{array}{l}
\varepsilon_{1} \\
\varepsilon_{2}
\end{array}\right](-\mathbf{z} \mid \Omega)=(-1)^{4 \varepsilon_{1} \cdot \varepsilon_{2}} \vartheta\left[\begin{array}{l}
\varepsilon_{1} \\
\varepsilon_{2}
\end{array}\right](\mathbf{z} \mid \Omega) .
$$

Therefore, we call a spin structure with characteristics $\left[\varepsilon_{1} \varepsilon_{2}\right]$ even or odd depending on whether $4 \varepsilon_{1} \cdot \varepsilon_{2}$ is even or odd. As we will see in Sect. 5 , the parity of a spin structure is related to the existence of zero-modes of the Dirac operator. A simple induction argument shows that there are $2^{g-1}\left(2^{g}-1\right)$ odd spin structures and $2^{g-1}\left(2^{g}+1\right)$ even ones. For example, in the one loop case, there is a single odd spin structure corresponding to $\vartheta\left[\begin{array}{c}\frac{1}{2} \\ \frac{1}{2}\end{array}\right](0 \mid \Omega)$, and three even spin structures with associated theta functions $\vartheta\left[\begin{array}{l}0 \\ 0\end{array}\right](0 \mid \Omega), \vartheta\left[\begin{array}{c}\frac{1}{2} \\ 0\end{array}\right](0 \mid \Omega), \vartheta\left[\begin{array}{l}0 \\ \frac{1}{2}\end{array}\right](0 \mid \Omega)$. In the two-loop

\footnotetext{
${ }^{2}$ Under a change of homology basis $\Delta$ shifts by an even half point, defined below
} 
case there are six odd spin structures associated to the theta functions

$$
\begin{aligned}
& \vartheta\left[\begin{array}{cc}
\frac{1}{2} & 0 \\
\frac{1}{2} & 0
\end{array}\right](0 \mid \Omega) \vartheta\left[\begin{array}{cc}
0 & \frac{1}{2} \\
0 & \frac{1}{2}
\end{array}\right](0 \mid \Omega) \vartheta\left[\begin{array}{cc}
\frac{1}{2} & 0 \\
\frac{1}{2} & \frac{1}{2}
\end{array}\right](0 \mid \Omega), \\
& \vartheta\left[\begin{array}{ll}
\frac{1}{2} & \frac{1}{2} \\
\frac{1}{2} & 0
\end{array}\right](0 \mid \Omega) \vartheta\left[\begin{array}{cc}
\frac{1}{2} & \frac{1}{2} \\
0 & \frac{1}{2}
\end{array}\right](0 \mid \Omega) \vartheta\left[\begin{array}{cc}
0 & \frac{1}{2} \\
\frac{1}{2} & \frac{1}{2}
\end{array}\right](0 \mid \Omega),
\end{aligned}
$$

and ten even ones with theta functions

$$
\begin{aligned}
& \vartheta\left[\begin{array}{ll}
\frac{1}{2} & 0 \\
0 & 0
\end{array}\right](0 \mid \Omega) \vartheta\left[\begin{array}{ll}
0 & 0 \\
\frac{1}{2} & 0
\end{array}\right](0 \mid \Omega) \vartheta\left[\begin{array}{ll}
0 & \frac{1}{2} \\
0 & 0
\end{array}\right](0 \mid \Omega) \\
& \vartheta\left[\begin{array}{ll}
0 & 0 \\
0 & \frac{1}{2}
\end{array}\right](0 \mid \Omega) \vartheta\left[\begin{array}{cc}
\frac{1}{2} & 0 \\
0 & \frac{1}{2}
\end{array}\right](0 \mid \Omega) \vartheta\left[\begin{array}{cc}
0 & \frac{1}{2} \\
\frac{1}{2} & 0
\end{array}\right](0 \mid \Omega) \\
& \vartheta\left[\begin{array}{ll}
\frac{1}{2} & \frac{1}{2} \\
0 & 0
\end{array}\right](0 \mid \Omega) \vartheta\left[\begin{array}{cc}
0 & 0 \\
\frac{1}{2} & \frac{1}{2}
\end{array}\right](0 \mid \Omega) \vartheta\left[\begin{array}{ll}
0 & 0 \\
0 & 0
\end{array}\right](0 \mid \Omega) \vartheta\left[\begin{array}{cc}
\frac{1}{2} & \frac{1}{2} \\
\frac{1}{2} & \frac{1}{2}
\end{array}\right](0 \mid \Omega) \text {. }
\end{aligned}
$$

Finally, we will see in Sect. 7 that modular transformations permute all the even and odd spin structures separately among themselves. This rather mathematical section will prove to be quite useful in our discussion of the Dirac determinant and of modular invariance.

\section{Holomorphic Factorization}

Quillen [15] has recently pointed out that functional determinants of operators on Riemann surfaces have interesting holomorphic properties. We will use these properties to investigate the Dirac determinant. However, holomorphic factorization has other important applications. For example, as pointed out in $[16,17,18]$ the very existence of chiral string theories relies on the existence of such holomorphic square roots.

In this section we will review the statement of Quillen's theorem and its relation to the geometry of determinant line bundles. We first give an heuristic Feynmandiagram proof of the theorem, and then proceed to the heat kernel proof of a slight generalization of the theorem. This generalization can also be understood in the context of determinant line bundles. In a beautiful recent paper [18] Belavin and Knizhnik have used similar methods to investigate the holomorphic properties of functional determinants on Teichmüller space. For completeness we extend the heuristic discussion of Quillen's theorem to rederive their results at the end of this section.

Abstractly, we will consider families of operators $D_{y}: L^{n} \rightarrow L^{n+2}$. If the parameter space $Y$ is a complex manifold, the $D_{y}$ can vary holomorphically with $y$. Quillen showed that in this case the determinant line bundle $\mathscr{L} \rightarrow Y$ can be given a holomorphic structure, and, if $D_{y}$ has no index, then the $\zeta$-regulated determinant satisfies

$$
\operatorname{det}_{\zeta} D^{\dagger} D=e^{-q}\left|\operatorname{det} D_{y}\right|^{2} \text {. }
$$

Here $\operatorname{det} D_{y}$ is a holomorphic function on $Y$. The "counterterm" $q$ is defined by choosing an operator $D_{0}$ to define the origin on $Y$ and setting

$$
q=\frac{i}{2 \pi} \int_{\Sigma} \operatorname{tr}\left(D-D_{0}\right)^{\dagger} \wedge\left(D-D_{0}\right) \text {. }
$$


The notation means that the family of operators is chosen so that any two differ by a one form. The trace is a finite dimensional trace over the fiber indices. Note that $\operatorname{det} D$ also depends on the choice of $D_{0}$.

More concretely, we can give a simple proof of Quillen's theorem using Feynman diagrams. Locally, $D_{y}$ looks like $\bar{\partial}+A$, so we can interpret $A$ as a gauge field. Since the obstruction to holomorphic factorization is local in $A$ we should be able to rederive (4.2) from perturbation theory. In perturbation theory

$$
W=\log \operatorname{det}_{\zeta} D^{\dagger} D
$$

is simply given by a sum of Feynman diagrams

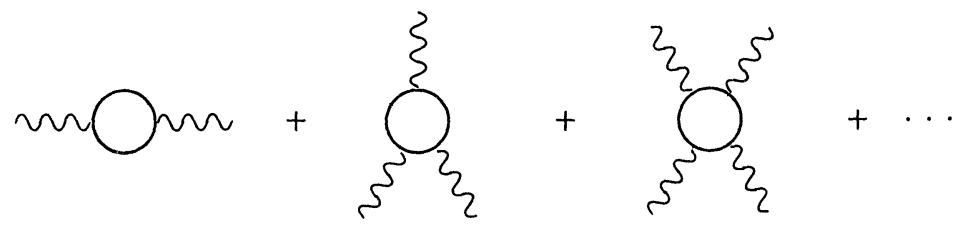

All diagrams but the first are convergent without regularization. Since the fields are massless their sum is a functional of the form $f\left[A_{z}\right]+\overline{f\left[A_{z}\right]}$. Thus their contribution to the determinant is gauge invariant and factorizes holomorphically as a functional of $A_{z}$. The situation is rather different for the vacuum polarization graph, which must be regulated. One can use Pauli-Villars regularization to maintain gauge invariance. Then as the PV mass goes to infinity one finds the nonholomorphic residue

$$
q=\frac{1}{2 \pi} \int_{\Sigma} d^{2} z A_{z} A_{\bar{z}} .
$$

It is more illuminating, however, to follow the two-dimensional calculation in [19]. From the axial anomaly we know that to order $A^{2}$ the contribution of a Weyl fermion to the vacuum functional is (in momentum space)

$$
\frac{1}{4 \pi} \int \frac{d^{2} p}{(2 \pi)^{2}} \frac{p_{z}}{p_{\bar{z}}} A_{\bar{z}}(p) A_{\bar{z}}(-p) \text {. }
$$

If we are computing $\operatorname{det} D^{\dagger} D$ we must add the other chirality. The result

$$
\frac{1}{4 \pi} \int \frac{d^{2} p}{(2 \pi)^{2}} \frac{p_{z}}{p_{\bar{z}}} A_{\bar{z}}(p) A_{\bar{z}}(-p)+\frac{1}{4 \pi} \int \frac{d^{2} p}{(2 \pi)^{2}} \frac{p_{\bar{z}}}{p_{z}} A_{z}(p) A_{z}(-p)
$$

continues to satisfy holomorphic factorization, but is not gauge invariant. We can restore gauge invariance - at the cost of factorization - by adding the counterterm

$$
\frac{1}{2 \pi} \int \frac{d^{2} p}{(2 \pi)^{2}} A_{z}(p) A_{\bar{z}}(-p)
$$

which is Quillen's counterterm [20].

In applications to string theory we will need to generalize (4.1)-(4.2) to the case when $D$ has an index, in order to handle the ghost operators. Therefore, we now give a heat kernel proof of the Quillen theorem in the case that $D_{y}$ has a nonzero 
index. We will simplify our calculation by assuming that $\operatorname{ker} D_{y}=0$ so that the cokernel has constant dimension. This is true for the ghost operators on higher loop Riemann surfaces. In any case the following analysis is easily generalized to the case when $D$ has a kernel [21].

We begin with the heat kernel definition of the determinant

$$
\log \operatorname{det} D^{\dagger} D=-\int_{\varepsilon}^{\infty} \frac{d t}{t} \operatorname{Tr} e^{-t D \dagger D}
$$

and, following Quillen, we take the second variation:

$$
\delta \bar{\delta} \operatorname{det} D^{\dagger} D=\delta \int_{\varepsilon}^{\infty} \operatorname{Tr} \bar{\delta} D^{\dagger} D e^{-t D^{\dagger} D} .
$$

Since $D$ has no kernel the right-hand side becomes simply

$$
=\delta \operatorname{Tr}\left(\bar{\delta} D^{\dagger} D \frac{1}{D^{\dagger} D} e^{-\varepsilon D^{\dagger} D}\right)
$$

which is

$$
\begin{gathered}
=\operatorname{Tr} \bar{\delta} D^{\dagger}\left(1-D \frac{1}{D^{\dagger} D} D^{\dagger}\right) \delta D \frac{1}{D^{\dagger} D} e^{-\varepsilon D^{\dagger} D} \\
-\varepsilon \int_{0}^{1} d s \operatorname{Tr} \bar{\delta} D^{\dagger} D \frac{1}{D^{\dagger} D} e^{-s \varepsilon D^{\dagger} D} D^{\dagger} \delta D e^{-(1-s) \varepsilon D^{\dagger} D} .
\end{gathered}
$$

Note that

$$
P=1-D \frac{1}{D^{\dagger} D} D^{\dagger}
$$

is the projector onto the kernel of $D^{\dagger}$. Thus the first trace in (4.11) is finite dimensional and we can take the limit $\varepsilon \rightarrow 0$. We can get a better understanding of this trace if we take a basis $\chi_{i}(y)$ for $\operatorname{ker} D_{y}^{\dagger}$ which varies antiholomorphically with $y$. (Such a basis exists since $D_{y}^{\dagger}$ varies antiholomorphically.) Then, a simple application of perturbation theory shows that

$$
\delta \bar{\delta} \log \operatorname{det}\left\langle\chi_{i} \mid \chi_{j}\right\rangle=\operatorname{Tr} P \delta D \frac{1}{D^{\dagger} D} \bar{\delta} D^{\dagger} .
$$

We can simplify the second trace by using

$$
e^{-s \varepsilon D^{\dagger} D} D^{\dagger}=D^{\dagger} e^{-s \varepsilon D D^{\dagger}}
$$

and applying (4.12). Since a finite dimensional trace is killed by the $\varepsilon \rightarrow 0$ limit, (4.11) becomes

$$
\delta_{y} \overline{\delta_{y}} \log \frac{\operatorname{det} D^{\dagger} D}{\operatorname{det}\left\langle\chi_{i} \mid \chi_{j}\right\rangle}=-\varepsilon \int_{0}^{1} d s \operatorname{Tr} \bar{\delta} D^{\dagger} e^{-s \varepsilon D D^{\dagger}} \delta D e^{-(1-s) \varepsilon D^{\dagger} D} .
$$

If $D_{y}$ has zero-modes we choose a basis $\phi_{i}$ varying holomorphically with $y$ and multiply $\operatorname{det}\left\langle\chi_{i} \mid \chi_{j}\right\rangle$ by $\operatorname{det}\left\langle\phi_{i} \mid \phi_{j}\right\rangle$ [21]. In the case considered by Quillen $\delta D=\delta A$ does not involve derivatives and Eq. (4.15) reduces to the simpler statement

$$
\delta \bar{\delta} \operatorname{det} D_{y}^{\dagger} D_{y}=-\varepsilon \operatorname{Tr} \bar{\delta} D^{\dagger} \delta D e^{-\varepsilon D^{\dagger} D} .
$$

Then, using the heat kernel expansion one recovers (4.1), (4.2). 
There is a third useful way to understand Quillen's theorem in terms of the geometry of determinant line bundles. If $D_{y}$ has no index then one can define a holomorphic section $\sigma$ of $\mathscr{L}$. The Hermitian norm

$$
\|\sigma\|^{2}=\operatorname{det}_{\zeta} D^{\dagger} D
$$

has a unique holomorphic compatible connection, whose curvature is just

$$
\frac{i}{2 \pi} \partial \bar{\partial} \log \|\sigma\|^{2} \text {. }
$$

On the other hand, Bismut and Freed [22] have computed this curvature in terms of the family index density. Using their formula one easily recovers (4.1) and (4.2).

The holomorphic factorization of determinants of operators with index can also be understood from the third point of view. We consider again a family with no kernel so that the index bundle

$$
\mathscr{L}=\left(\Lambda^{\max } \operatorname{ker} D_{y}\right)^{*} \otimes\left(\Lambda^{\max } \operatorname{cok} D_{y}\right)
$$

is well-defined as it stands. We can define a section of $\mathscr{L}$ which is holomorphic in the holomorphic structure defined by Quillen. Choose a point $y$ in some neighborhood of $y_{0}$ and take

$$
\sigma(y)=\frac{\operatorname{det}\left\langle\chi_{i}(y) \mid \chi_{j}\left(y_{0}\right)\right\rangle}{\operatorname{det}\left\langle\chi_{i}(y) \mid \chi_{j}(y)\right\rangle} \chi_{1}(y) \wedge \ldots \wedge \chi_{k}(y) .
$$

We define the neighborhood on which (4.20) holds to be the set of points $y$ such that

$$
\operatorname{det}\left\langle\chi_{i}(y) \mid \chi_{j}\left(y_{0}\right)\right\rangle \neq 0 \text {. }
$$

We can extend $\sigma$ to the rest of $Y$ by taking $\sigma=0$ outside the neighborhood. This is a holomorphic section of $\mathscr{L}$ [22]. The Quillen norm of this section is just

$$
\|\sigma\|^{2}=\left|\operatorname{det}\left\langle\chi_{i}(y) \mid \chi_{j}\left(y_{0}\right)\right\rangle\right|^{2}\left(\frac{\operatorname{det} D^{\dagger} D}{\operatorname{det}\left\langle\chi_{i}(y) \mid \chi_{j}(y)\right\rangle}\right) .
$$

On the other hand, the curvature of (4.7) has been computed by Bismut and Freed in terms of the family index density. Thus if we choose the basis $\chi_{i}(y)$ to vary antiholomorphically, then the obstruction to the holomorphic factorization of

$$
\frac{\operatorname{det} D_{y}^{\dagger} D_{y}}{\operatorname{det}\left\langle\chi_{i}(y) \mid \chi_{j}(y)\right\rangle}
$$

is again just given by the family index density.

In the following section we will apply Quillen's theorem to families of operators over the Picard variety. For completeness we describe here a recent result of Belavin and Knizhnik who considered families of operators over moduli space. In [18] a heat kernel or index bundle approach was used. We show here how the result can be understood as the gravitational analog of the Feynman diagram argument we used before. We begin by defining

$$
W[\hat{g}] \equiv \log \left(\frac{\operatorname{det}^{\prime} D^{\dagger} D}{\operatorname{det}\left\langle\chi_{i} \mid \chi_{j}\right\rangle \operatorname{det}\left\langle\phi_{i} \mid \phi_{j}\right\rangle}\right),
$$


where $D$ is now the operator $\nabla_{w}^{n}$ of (3.10), and $e^{W}$ is the partition function for a pair of spin $\pm \frac{n}{2}$ particles on the worldsheet. Following [18], we consider metric deformations $g+\delta g$ which look like

$$
e^{2 \phi}|d z+\eta d \bar{z}|^{2},
$$

where $\eta$ is a (small) Beltrami differential. From the family index theorem or from the general heat kernel formula (4.15), we know that the obstruction to holomorphic factorization (in $\eta$ and $\bar{\eta}$, i.e. on Teichmüller space) is local so it suffices to consider Beltrami differentials with support in a coordinate patch, $\mathscr{U}$. These will not correspond to Teichmüller deformations, but the final result will apply to such deformations. Using the conformal anomaly we can write

$$
\begin{aligned}
W[g+\delta g] & =W[\hat{g}]+\frac{3(n+1)^{2}-1}{24 \pi} S_{L}[\phi, \hat{g}] \\
& =W[\hat{g}]+\frac{3(n+1)^{2}-1}{24 \pi} \int_{\Sigma} d^{2} \sigma \sqrt{\hat{g}}\left(\hat{g}^{a b} \partial_{a} \phi \partial_{b} \phi-\hat{R} \phi\right),
\end{aligned}
$$

where $\hat{g}$ is just $|d z+\eta d \bar{z}|^{2}$ in $\mathscr{U}$, and (4.25) defines the Liouville action $S_{L}$. Thus $\hat{g}$ is a small deviation from a flat metric in $\mathscr{U}$, and we can use perturbation theory to find the part in $W[\hat{g}]$ of second order in $\eta$. We now use Orlando Alvarez's trick [16] to write the appropriate perturbation theory by considering the operator $D: L^{n}$ $\rightarrow L^{n+2}$ as a chiral Dirac operator coupled to the vector bundle $L^{n+1}$. Thus the effective action $W$ is, to second order in $\eta$, just given by the gravitational contribution computed in [19] and the gauge contribution of (4.6) with $A=\frac{n+1}{2} \omega$, where $\omega$ is the Riemannian spin connection. That is

$$
\begin{aligned}
W^{(2)}[\hat{g}]= & -\frac{1}{192 \pi} \int \frac{d^{2} p}{(2 \pi)^{2}} \frac{p_{z}^{3}}{p_{\bar{z}}} \hat{g}_{\bar{z} \bar{z}}(p) \hat{g}_{\bar{z} \bar{z}}(-p)-\frac{1}{192 \pi} \int \frac{d^{2} p}{(2 \pi)^{2}} \frac{p_{\bar{z}}^{3}}{p_{z}} \hat{g}_{z z}(p) \hat{g}_{z z}(-p) \\
& +\frac{1}{4 \pi} \int \frac{d^{2} p}{(2 \pi)^{2}} \frac{p_{z}}{p_{\bar{z}}} A_{\bar{z}}(p) A_{\bar{z}}(-p)+\frac{1}{4 \pi} \int \frac{d^{2} p}{(2 \pi)^{2}} \frac{p_{\bar{z}}}{p_{z}} A_{z}(p) A_{z}(-p)
\end{aligned}
$$

Substituting for the gauge field and adding the counterterms to restore coordinate invariance (as described in [19]) we obtain the famous result

$$
W^{(2)}[\hat{g}]=\frac{3(n+1)^{2}-1}{192 \pi} \int \frac{d^{2} p}{(2 \pi)^{2}} \frac{\hat{R}(p) \hat{R}(-p)}{p_{z} p_{\bar{z}}} .
$$

The coefficient in front of the integral is just that of the usual conformal anomaly in our conventions. Finally, one computes the curvature for the metric $|d z+\eta d z|^{2}$

$$
\hat{R}=-\partial_{z}^{2} \bar{\eta}-\partial_{\bar{z}}^{2} \eta+2 \partial_{z} \partial_{\bar{z}}(\eta \bar{\eta})+\frac{1}{2}\left[\partial_{z} \eta \partial_{\bar{z}} \bar{\eta}-\partial_{z} \bar{\eta} \partial_{\bar{z}} \eta\right] .
$$

Combining (4.25), (4.27), and (4.28) we find

$$
\begin{aligned}
\delta_{\eta} \delta_{\bar{\eta}} W= & \frac{3(n+1)^{2}-1}{96 \pi} \int d^{2} \sigma\left[\partial_{z} \eta \partial_{\bar{z}} \bar{\eta}+2\left(\partial_{z} \eta \bar{\eta} \partial_{z} \phi+\partial_{z} \bar{\eta} \eta \partial_{\bar{z}} \phi\right)\right. \\
& \left.+4 \eta \bar{\eta} \partial_{\bar{z}} \phi \partial_{\bar{z}} \phi-6 \eta \bar{\eta} \partial_{z} \partial_{\bar{z}} \phi\right],
\end{aligned}
$$


and in terms of the original metric deformations $\delta g_{w w}$ this is simply

$$
\frac{3(n+1)^{2}-1}{96 \pi}\left[\int d^{2} \sigma \sqrt{g}\left(g^{z \bar{z}}\right)^{3} \nabla_{z}^{-2} \delta g_{\bar{z} \bar{z}} \nabla_{2}^{z} \delta g_{z z}-\frac{3}{2} \int d^{2} \sigma \sqrt{g}\left(g^{z \bar{z}}\right)^{2} \delta g_{z z} \delta g_{\bar{z} \bar{z}} R\right],
$$

reproducing the result in [18].

The Belavin-Knizhnik obstruction can be rewritten in terms of the WeilPetersson Kähler potential on Teichmüller space [23]. Recall that Teichmüller space $T$ can be represented as the space of constant curvature metrics: namely, for any metric $g$ there is Weyl transformation so that $\hat{g}=e^{-2 \phi} g$ is a constant curvature metric. ${ }^{3}$ It therefore suffices to consider the holomorphic obstruction on $T$. In the standard Bers embedding of $T$ as a bounded domain in $C^{3 g-3}$ the holomorphic cotangent space of $T$ is identified with the integrable holomorphic quadratic differentials [24]. For these deformations the first term in (4.30) is zero so

$$
\delta \bar{\delta} W=-\frac{3(n+1)^{2}-1}{64 \pi}\langle\delta g, \delta g\rangle_{\mathrm{WP}}
$$

where $\langle,\rangle_{\mathrm{WP}}$ is the Weil-Petersson metric. Since the Weil-Petersson metric is Kähler [24] and the Bers embedding provides globally defined analytic coordinates on $T$, there must be a globally defined Kähler potential $U_{\text {WP. }}$. It follows that the complete obstruction to holomorphic factorization of (4.22) is given by

$$
\exp \frac{3(n+1)^{2}-1}{24 \pi}\left(S_{L}[\phi, \hat{g}]-\frac{3}{8} U_{\mathrm{WP}}\right) \text {. }
$$

Note that $S_{L}$ and $U_{\mathrm{WP}}$ themselves make no reference to the spin $n$. Thus the product of a collection of determinants of spins $n_{i}$ will have a total obstruction proportional to $\sum_{i}\left[3\left(n_{i}+1\right)^{2}-1\right]$. Therefore, as Belavin and Knizhnik observed, when the conformal anomaly cancels the string integrand is the square of a holomorphic function on Teichmüller space (even though the individual determinants are not). We believe this result is very powerful and should allow one to apply arguments similar to those used in the next section to obtain useful expressions for the functional determinants appearing in string theory in terms of "canonical" or "natural" functions associated to a Riemann surface.

\section{Chiral Dirac Determinants}

In this section we will apply the Quillen theorem to a family of Dirac operators over the Picard variety, which is essentially the original context considered by Quillen. Since the Dirac operator has no index the theorem will be especially easy to apply. We will begin by considering the family of operators in (3.16) with $\mathbf{u} \in C^{g}$ (or, if we wish to consider a twisted family of operators we may take $\mathbf{u} \in J(\Sigma)$ ). A simple argument using the Quillen theorem and gauge invariance shows that $\operatorname{det} D(\mathbf{u})$ is proportional to a theta function, but the argument is not strong enough

\footnotetext{
${ }^{3}$ In general $g$ will also have to be pulled back by a diffeomorphism, but this complication is irrelevant for our purposes. Note too that for the torus we can take $R=0$. That is why explicit calculations on the torus do not encounter this obstruction
} 
to determine the characteristics. Thus we give a second independent argument using the methods of Sect. 3d. The idea is simple: Since the Dirac operator has no index, $D(\mathbf{u})$ generically has no zero-modes. In other words, $\operatorname{det} D(\mathbf{u})$ will vanish in $J(\Sigma)$ on a subset of complex codimension 1. By choosing the spin structure judiciously, we will show that the locus of zeroes of $\operatorname{det} D(\mathbf{u})$ is the $\Theta$-divisor described in Sect. 3. At the end of this section we comment on the ghost operators.

If we apply (4.1) and (4.2) to the $U(1)$ gauge field (3.15) parametrized by the Jacobian, then, using (3.7), (3.8), (3.15) and making a trivial redefinition of $q$ in (4.2), we find:

$$
\operatorname{det} D(\mathbf{u})^{\dagger} D(\mathbf{u})=e^{i \pi(u-\bar{u}) \cdot(\Omega-\bar{\Omega})^{-1} \cdot(u-\bar{u})}|g(\mathbf{u})|^{2} .
$$

Notice now that shifts of $\mathbf{u}$ by the Jacobian lattice $\mathbf{u} \rightarrow \mathbf{u}+\mathbf{n}+\Omega \mathbf{m}$ are equivalent to well-defined $U(1)$ gauge transformations on $\Sigma$ given by

$$
\mathscr{U}(P)=\exp -2 \pi i\left(\mathbf{m} \cdot \int_{P_{0}}^{P} \boldsymbol{\alpha}-\mathbf{n} \cdot \int_{P_{0}}^{P} \boldsymbol{\beta}\right) .
$$

Since the determinant (5.1) was computed by means of a gauge-invariant $\zeta$-function regulator it must necessarily be gauge invariant under these gauge transformations. Since the exponent in (5.1) is not gauge invariant we can deduce the transformation properties of $g(\mathbf{u})$ under lattice shifts,

$$
\begin{aligned}
g(\mathbf{u}+\mathbf{n}) & =e^{i \phi(\mathbf{n})} g(\mathbf{u}), \\
g(\mathbf{u}+\Omega \mathbf{m}) & =e^{i \psi(\mathbf{m})} e^{-i \pi \mathbf{m} \cdot \Omega \cdot \mathbf{m}-2 \pi i \mathbf{m} \cdot \mathbf{u}} g(\mathbf{u}),
\end{aligned}
$$

and since the phases $e^{i \phi(\mathbf{n})}$ and $e^{i \psi(\mathbf{m})}$ provide a unitary representation of $Z^{g}$, they must be of the form $e^{i \phi(\mathbf{n})}=e^{2 \pi i \mathbf{n} \cdot \mathbf{a}}, e^{i \psi(\mathbf{m})}=e^{-2 \pi i \mathbf{m} \cdot \mathbf{b}}$. Using a holomorphic Fourier decomposition of $g(\mathbf{u})$ we see that (5.3) uniquely defines a $\vartheta$-function up to a constant. Hence, by (3.17),

$$
g(\mathbf{u})=\operatorname{const} \vartheta\left[\begin{array}{l}
\mathbf{a} \\
\mathbf{b}
\end{array}\right](\mathbf{u} \mid \Omega)
$$

for some real characteristics $\mathbf{a}, \mathbf{b}$.

We have not used any information about the spin structure to derive (5.4), so it is not unreasonable that the characteristics $\mathbf{a}, \mathbf{b}$ remain undetermined. We now use the methods of Sect. 3d to compute a, b. Quillen's construction of the chiral Dirac determinant shows that it is a holomorphic section of a holomorphic line bundle $\mathscr{L}$ on $J(\Sigma)$ (note that $\operatorname{det} D^{\dagger} D$ does not blow up for any $\mathbf{u}$ ). From the family index theorem and (3.21) we know that $\mathscr{L}$ is the line bundle of a theta function, and therefore by the comments following (3.21) that bundle admits a single holomorphic section. In order to determine this section, we simply compute its divisor. The divisor of the determinant consists of those points $\mathbf{u}$ in $J(\Sigma)$ where $D(\mathbf{u})$ has a zero mode. By the arguments preceding $(3.15), D(\mathbf{u})$ has a zero mode whenever the line bundle $L_{\alpha}^{-1} \otimes V(\mathbf{u})$ has a holomorphic section. We can determine those bundles admitting a holomorphic section by studying the divisor of $L_{\alpha}^{-1} \otimes V(\mathbf{u})$. Let $A_{\alpha}$ be a divisor of $L_{\alpha}^{-1}$. If $d_{\mathbf{u}}$ is the divisor of $V(\mathbf{u})$, then $L_{\alpha}^{-1} \otimes V(\mathbf{u})$ has divisor 
$A_{\alpha}+d_{\mathbf{u}}$. We can write $d_{\mathbf{u}}$ explicitly in terms of $\mathbf{u}$ as follows. The function

$$
f_{\mathbf{u}}(P)=\frac{\vartheta\left[\begin{array}{l}
\theta \\
\phi
\end{array}\right]\left(\mathbf{z}+\int_{P_{0}}^{P} \omega \mid \Omega\right)}{\vartheta\left[\begin{array}{l}
0 \\
0
\end{array}\right]\left(\mathbf{z}+\int_{P_{0}}^{P} \omega \mid \Omega\right)}
$$

is meromorphic and has periodicities

$$
\Delta_{n \cdot a} f_{u}=e^{2 \pi i n \cdot \theta} f_{u}, \quad \Delta_{m \cdot b} f_{u}=e^{-2 \pi i m \cdot \phi} f_{u}
$$

around the $\mathbf{a}$ and $\mathbf{b}$ cycles respectively. ( $\mathbf{z}$ is fixed and arbitrary.) Thus $f_{\mathbf{u}}(P)$ is a meromorphic section of $V(\mathbf{u})$. By the Riemann vanishing theorem $f_{\mathbf{u}}(P)$ has poles at $g$ points $Q_{i}$ satisfying

$$
\mathbf{z}+\sum_{i=1}^{g} \int_{P_{0}}^{Q_{i}} \boldsymbol{\omega}=\Delta,
$$

and zeros at $g$ points $R_{i}$ such that

$$
\mathbf{z}+\mathbf{u}+\sum_{i=1}^{g} \int_{P_{0}}^{R_{i}} \boldsymbol{\omega}=\boldsymbol{\Delta},
$$

hence

$$
\mathbf{u}=-\sum_{i=1}^{g}\left(\int_{P_{0}}^{R_{i}} \boldsymbol{\omega}-\int_{P_{0}}^{Q_{i}} \boldsymbol{\omega}\right)=-I\left[\sum_{1}^{g} R_{i}-\sum_{1}^{g} Q_{i}\right] .
$$

Now recall that $L_{\alpha}^{-1} \otimes V$ has a holomorphic section if and only if it has a divisor of the form

$$
A_{\alpha}+d_{\mathbf{u}}=\sum_{i=1}^{g-1} P_{i} .
$$

Choosing an arbitrary point $P_{0}$ on $\Sigma$, (5.7) can be rewritten as

$$
d_{\mathbf{u}}=(g-1) P_{0}-A_{\alpha}+\left(\sum_{i=1}^{g-1} P_{i}-(g-1) P_{0}\right),
$$

and using (5.6) we have:

$$
-\mathbf{u}=I\left[(g-1) P_{0}-A_{\alpha}\right]+I\left[\sum_{1}^{g-1} P_{i}-(g-1) P_{0}\right] .
$$

From (3.28) there is a spin structure $D_{0}$ such that the first term on the right-hand side of (5.9) is just the vector of Riemann constants. Thus

$$
\mathbf{u}=\boldsymbol{\Delta}-\sum_{i=1}^{g-1} \int_{P_{0}}^{P_{i}} \boldsymbol{\omega}
$$

Using the characterization (3.25) of the $\Theta$-divisor, we conclude that for the spin structure $D_{0}$, the characteristics $\mathbf{a}, \mathbf{b}$ in (5.4) are equal to zero. It follows that the determinant is given by

$$
\operatorname{det} D(\mathbf{u})^{\dagger} D(\mathbf{u})=|c|^{2} e^{i \pi(u-\tilde{u}) \cdot(\Omega-\bar{\Omega})^{-1} \cdot(u-\bar{u})}|\vartheta(\mathbf{u} \mid \Omega)|^{2} .
$$

The overall positive constant $|c|^{2}$ is a function only of the metric, not of the twisting. 
In order to define the chiral determinant we must take the square root of (5.11). There are many ways to define chiral square roots, and we must choose a definition suitable to the physical problem under study. In the context of string theories we would like to separate the contributions of left-movers from right-movers. In Sect. 2 we found that the left- and right-movers contribute holomorphic and antiholomorphic functions of the moduli. Furthermore, from the formula for variation of a covariant derivative under a traceless deformation of the metric [25],

$$
\delta \nabla_{w}^{n}=-\frac{1}{2} \delta g_{w w} \nabla^{w}+\frac{n}{2} \nabla^{w}\left(\delta g_{w w}\right),
$$

we see that naively we expect $\operatorname{det} \nabla_{w}^{n}$ to be a holomorphic function on Teichmüller space [26]. Therefore, we will proceed with the hypothesis that on higher genus surfaces the contributions of left- and right-movers can be distinguished by holomorphy in the moduli. In this context the work of Belavin and Knizhnik shows that the quantity in (4.32) is the obstruction to the decoupling of left-movers from right-movers. Since the $|c|^{2}$ is, up to the obstruction (4.32), the square of a holomorphic function on moduli space, we sacrifice holomorphy on the Picard variety and write:

$$
u-\bar{u}-(\Omega-\bar{\Omega}) \boldsymbol{\theta},
$$

so that

$$
\operatorname{det} D^{\dagger}(\mathbf{u}) D(\mathbf{u})=|c|^{2}\left|e^{i \pi \theta \cdot \Omega \cdot \theta} \vartheta\left[\begin{array}{l}
0 \\
0
\end{array}\right](\mathbf{u} \mid \Omega)\right|^{2}=|c|^{2}\left|\vartheta\left[\begin{array}{l}
\boldsymbol{\theta} \\
\boldsymbol{\phi}
\end{array}\right](0 \mid \Omega) e^{-2 \pi i \theta \cdot \phi}\right|^{2} .
$$

Therefore, we have

$$
\operatorname{det} D(\mathbf{u})=c \vartheta\left[\begin{array}{l}
\boldsymbol{\theta} \\
\boldsymbol{\phi}
\end{array}\right](0 \mid \Omega) .
$$

Actually the left-hand side of (5.13) is not purely a function of the moduli because of conformal and diffeomorphism anomalies. If we represent the metrics by $g=f^{*} e^{2 \phi} \hat{g}$, then the modulus and phase of $c$ will depend on $\phi$ and $f$ respectively. In applications to string theory we may be cavalier about the anomalies since we know that the combination of determinants in the string path integral is anomaly free. Thus we can consider $c$ as a function only of the moduli.

As a simple application, (5.12) makes transparent some well-known theorems on the existence of harmonic spinors. For example from (5.12) we learn that all odd spin structures have at least one zero-mode while the even spin structures generically have no zero modes. These results are derived in [27, 28]. Furthermore, it is known that for genus two the even theta functions do not vanish at $z=0$ except when $\Omega$ is diagonal [13]. (This corresponds to a degenerate curve for which the handles have been pulled off to infinity.) For genus three and larger there are period matrices and even characteristics such that $\vartheta(\mathbf{z}=0)=0$ [13], so that the dimension of the space of harmonic spinors is constant for $g=2$ and varies with the complex structure for $g \geqq 3$. These results were derived by Hitchin in 1974 [29]. 
We can also apply the Quillen theorem to the fermionic ghost operator of string theory, namely

$$
\nabla_{w}^{+1}: L_{\alpha} \otimes V \rightarrow L_{\alpha}^{3} \otimes V
$$

One finds that if $\chi_{i}$ is a basis of $\operatorname{ker}_{3}^{w}$ (that is, a basis of supermoduli) varying holomorphically with $\bar{u}$ then

$$
\frac{\operatorname{det}(\nabla(\mathbf{u}))^{\dagger} \nabla(\mathbf{u})}{\operatorname{det}\left\langle\chi_{i} \mid \chi_{j}\right\rangle}=\left|c^{\prime}\right|^{2}\left|e^{i \pi \theta \cdot \Omega \cdot \theta}\right|^{2}|g(\mathbf{u})|^{2},
$$

where $\left|c^{\prime}\right|^{2}$ is independent of $\mathbf{u}$, and $g(\mathbf{u})$ is holomorphic. Unfortunately, there are many choices of holomorphically varying bases, so gauge invariance is not as powerful in this case. Therefore we will simply make a few remarks. First, note that there is no holomorphically varying basis $\chi_{i}$ which changes by a unitary transformation under a shift $\mathbf{u} \rightarrow \mathbf{u}+\mathbf{m}+\Omega \mathbf{n}$. For, if there were, gauge invariance would imply that $g(\mathbf{u})$ is a theta function, which must vanish on a subvariety of $J(\Sigma)$. On the other hand, the left-hand side of (5.15) never vanishes. In fact, we could choose our basis so that $g(\mathbf{u})=1$. Alternatively, using the Riemann vanishing theorem, it is possible to write a choice of basis $\chi_{i}$ for which the $\mathbf{u}$-dependence is explicit. One finds in this case that $g(\mathbf{u})$ can be expressed in terms of theta functions of weight $l=2 g-2$ ( $=$ the number of supermoduli). However the theta functions of weight $l$ form a vector space of dimension $l^{g}$ so that we do not learn much. Although the combination (5.15) is basis-dependent, the superstring path-integral measure is in fact basis-independent (see Sect. 7). An astute choice of basis should lead to important simplifications.

\section{Bosonization}

Bosonization is an important aspect of two-dimensional field theory. Although the local theory of bosonization on higher genus Riemann surfaces has been worked out [3], no complete treatment yet exists. We now show that the results of Sect. 5 can be used to give a prescription for the lattice sum needed in bosonization of spin- $\frac{1}{2}$ particles. In particular we use bosonization to evaluate $|c|^{2}$, where $c$ is the function of the metric defined in (5.13). Furthermore, we will see that non-chiral bosonization involves an interesting subtlety. Since, roughly speaking, a Bose theory doesn't "know" about a spin structure, it will turn out that a Bose theory corresponds to an average over spin structures of Fermi theories. We consider first nonchiral bosonization to avoid subtleties peculiar to left-moving bosons. This is the reason we only evaluate the absolute square $|c|^{2}$. Then using the remarks of Sect. 5, we can separate out the contribution of the left-movers and give a prescription for the lattice contribution in chiral bosonization.

As we saw in Sect. 2, bosonization on compact spaces involves a sum over soliton sectors. If we formulate the $S O(n)$ current algebra on the cylinder we will have $n$ scalar fields which can have shifts by the integral or half-integral vectors in the weight lattice of $S O(n)$. Furthermore, on the torus or the cylinder we see that twisted fermions correspond under bosonization to scalars which shift by a translate of the $S O(n)$ weight lattice. We now generalize these notions to higher 
loops, taking $n=2$ for simplicity. Therefore, consider a real Bose field $x$ on a Riemann surface $\Sigma$. We can introduce soliton sectors into the theory if we allow $x$ to have shifts around the nontrivial homology cycles. Therefore we can define the $\left(n^{1}, \ldots, n^{g}, m^{1}, \ldots, m^{g}\right)$-soliton sector by requiring

$$
\begin{aligned}
& x \rightarrow x+\left(n^{i}+\theta^{i}\right) \text { around } a_{i}, \\
& x \rightarrow x+\left(m^{i}+\phi^{i}\right) \text { around } b_{i},
\end{aligned}
$$

where $0 \leqq \theta^{i}, \phi^{i} \leqq 1$ and $n^{i}, m^{i} \in \frac{1}{2} Z, i=1, \ldots, g$. We can simplify the derivation by first summing over soliton sectors with $n, m$ integral, and then summing over halfintegral shifts in $\theta, \phi$. The soliton solution is given by

$$
d x_{n, m}=\sum_{1}^{g}(n+\theta)^{i} \alpha_{i}+\sum_{1}^{g}(m+\phi)^{i} \beta_{i},
$$

where $\alpha_{i}, \beta_{i}$ are the harmonic forms of Sect. 3. We can express the action for the soliton:

$$
S[x]=2 \pi \int d x \wedge * d x
$$

in terms of the matrices

$$
A_{i j}=\int \alpha^{i} \wedge * \alpha^{j}, \quad B_{i j}=\int \beta^{i} \wedge * \beta^{j}, \quad C_{i j}=\int \alpha^{i} \wedge * \beta^{j} .
$$

Using Sect. 3 one finds that the period matrix is simply given in terms of these matrices by

$$
\Omega=B^{-1} C^{T}+i B^{-1} .
$$

With the soliton action one can then write the partition function for the theory:

$$
Z=\left(\frac{\operatorname{det}^{\prime}-\nabla^{2}}{\int \sqrt{g}}\right)^{-1 / 2} \sum_{\mathbf{n}, \mathbf{m} \in Z^{8}} e^{-S_{n, m}}
$$

where $S_{n, m}$ is the action for the soliton (6.2). We will now relate this sum to theta functions, thereby establishing a link with fermionic partition functions.

Holding $\mathbf{n}$ fixed, we use (6.3)-(6.5) and apply the Poisson summation formula to the sum on $\mathbf{m}$ to obtain:

$$
Z=\left(\frac{\operatorname{det}^{\prime}-\nabla^{2}}{\int \sqrt{g} \operatorname{det} \operatorname{Im} \Omega}\right)^{-1 / 2} \frac{1}{2^{g}} \sum_{n, m} e^{\mathscr{A} \ell_{n, m}} e^{\overline{\mathscr{A}_{n, m}}},
$$

where $\mathscr{A}_{n, m}$ is a complex number. Explicitly one finds, using (6.2), (6.4), (6.5), and (6.7),

$$
\begin{aligned}
& \mathscr{A}_{n, m}=i \pi\left(\mathbf{n}+\boldsymbol{\theta}+\frac{1}{2} \mathbf{m}\right) \cdot \Omega \cdot\left(\mathbf{n}+\boldsymbol{\theta}+\frac{1}{2} \mathbf{m}\right)+2 \pi i\left(\mathbf{n}+\boldsymbol{\theta}+\frac{1}{2} \mathbf{m}\right) \cdot \boldsymbol{\phi}, \\
& \overline{\mathscr{A}}_{n, m}=-i \pi\left(\mathbf{n}+\boldsymbol{\theta}-\frac{1}{2} \mathbf{m}\right) \cdot \bar{\Omega} \cdot\left(\mathbf{n}+\boldsymbol{\theta}-\frac{1}{2} \mathbf{m}\right)-2 \pi i\left(\mathbf{n}+\boldsymbol{\theta}-\frac{1}{2} \mathbf{m}\right) \cdot \boldsymbol{\phi} .
\end{aligned}
$$

We can express the sum (6.7) in terms of theta functions. The result is:

$$
Z=\left(\frac{\operatorname{det}^{\prime}-\nabla^{2}}{\int \sqrt{g} \operatorname{det} \operatorname{Im} \Omega}\right)^{-1 / 2} \frac{1}{2^{g}} \sum_{\substack{\varepsilon_{1} \in Z_{2}^{g} \\
\varepsilon_{2} \in Z_{2}^{g}}}\left|\vartheta\left[\begin{array}{r}
\boldsymbol{\theta}+\boldsymbol{\varepsilon}_{1} \\
-\boldsymbol{\phi}+\boldsymbol{\varepsilon}_{2}
\end{array}\right](0 \mid \Omega)\right|^{2} e^{4 \pi i \varepsilon_{1} \cdot \varepsilon_{2}} .
$$


Now we include the full weight lattice, allowing soliton sectors with $n^{i}, m^{i}$ integral and half-integral. The partition function then becomes

$$
Z=|c|^{2} \sum_{\varepsilon_{1}, \varepsilon_{2} \in Z_{2}^{g}}\left|\vartheta\left[\begin{array}{c}
\boldsymbol{\theta}+\varepsilon_{1} \\
\phi+\varepsilon_{2}
\end{array}\right](0 \mid \Omega)\right|^{2} .
$$

Therefore, this Bose theory corresponds to a sum over all the $2^{2 g}$ Fermi theories on $\Sigma$. Weighted sums over subsets of the lattice correspond to particular spin structures, suggesting the possibility of bosonizing a Fermi theory with a single spin structure. Comparing the overall normalization of the partition functions we learn that the function $|c|^{2}$ of the last section is given by

$$
|c|^{2}=\operatorname{const}\left(\frac{\operatorname{det}^{\prime}-\nabla^{2}}{\int \sqrt{g} \operatorname{det} \operatorname{Im} \Omega}\right)^{-1 / 2}
$$

up to some numerical constant. This derivation is not rigorous, but (6.11) does pass two important consistency checks. First, both sides of the equation have the same conformal anomaly. Second, in the case of the torus one can easily compute both quantities directly. For the metric $\left|d \sigma_{1}+\tau d \sigma_{2}\right|^{2}$ one finds the right-hand side is given by

$$
\frac{1}{2|\eta(\tau)|^{2}}
$$

which agrees with (2.3) and (5.13). Thus (6.11) is also consistent with the naive factorization of determinants in the limit when a handle is pulled off to infinity.

We now turn to the case of chiral bosonization. We see from (6.7) that the contributions of the various soliton sectors are holomorphic squares. In the natural complex structure on Teichmüller space the period matrix is a holomorphic function of the moduli [24]. Therefore, according to the identification of Sect. $5, \mathscr{A}_{n, m}$ should be thought of as the action of a right-moving soliton. Thus, the expression (6.10) allows a separation of the contributions of left-movers and rightmovers, which we identify as holomorphic and anti-holomorphic functions of the moduli. If we simply drop the contribution of the left- or right-movers we should obtain the partition function for a chiral boson. Using bosonization arguments such as these one can verify that the contribution of the left moving $E_{8} \times E_{8}$ solitons to the string path integral is

$$
\left(c^{8} \sum_{\varepsilon_{1}, \varepsilon_{2}}\left(\vartheta\left[\begin{array}{l}
\boldsymbol{\varepsilon}_{1} \\
\boldsymbol{\varepsilon}_{2}
\end{array}\right](0 \mid \Omega)\right)^{8}\right)^{2}
$$

as expected from the fermionic formulation of the current algebra. In string theory we also need to consider the ghosts. For these the bosonization is more subtle [3] and the sum over soliton sectors seems to yield rather awkward expressions.

\section{Modular Invariance in the Heterotic String}

In the previous sections we have been able to compute the spin-structure dependence of the determinant of the chiral Dirac operator on Riemann surfaces. 
In this section, we will apply those results to discuss some aspects of the higher loop heterotic string amplitudes. In particular, as a first application, we will verify some results of Witten on global anomalies [30]. It has recently been pointed out that two-dimensional anomaly cancellation does not completely fix the heterotic string amplitudes [31, 32]. As a second application, we will discuss this ambiguity from the point of view of modular orbits of theta characteristics.

We begin by presenting an expression for the higher loop vacuum to vacuum amplitudes in the heterotic string. At $g$-loops this amplitude can be derived from a functional integral over two-dimensional supergeometries of a $g$-handled Riemann surface $\Sigma$, and over quantum matter fields living on this surface [2]. For the heterotic string a supergeometry on a Riemann surface is specified by a Riemannian metric (a background graviton field configuration) together with a section of $L_{\alpha}^{-3}$ (a background gravitino field configuration) [33]. The matter fields may be described as follows. There are 10 left- and right-moving bosonic coordinates describing the embedding of $\Sigma$ onto the world surface of the string in 10-dimensional spacetime. Spin waves on the world surface are described by the $S O(10)$ current algebra constructed from the 10 right-moving spinor sections of $L_{\alpha}$ familiar from the NSR model. ${ }^{4}$ In addition, heterotic strings have gauge currents. In the $O(32)$ string these currents can be constructed from 32 left-moving spinor sections of $L_{\beta}^{-1}$. The spin structures $\alpha, \beta$ are independent. In the $E_{8} \times E_{8}$ string the 32 gauge fermions are split into two sets of 16 , each with independent spin structures $\beta$ and $\gamma$. In Minkowski space the spinors are Majorana-Weyl, but in Euclidean space they must be Weyl. The Euclidean path integral quantization of a Majorana-Weyl spinor introduces a Pfaffian of the complex chiral Dirac operator which results is an extra square root of the relevant functional determinant.

Gauge-fixing the symmetries of the Euclidean heterotic string action reduces the infinite-dimensional functional integral to a finite-dimensional integral over the moduli space of $\Sigma$ which describes gauge-inequivalent graviton field configurations [34-37] together with a finite dimensional Grassmann integral over supermoduli space which describes gauge-inequivalent background gravitino field configurations $[3,17,38]$. The integration over moduli space is expressed as an integration over a fundamental domain $F$ of the Teichmüller space $T$ under the action of $\Omega(\Sigma)[39,35]$. In addition, in order to define a physically reasonable string theory one must include projections onto sectors with even fermion number [40]. This is accomplished in the path integral formulation by summing over the independent spin structures for the two sets of fermions ${ }^{5}$.

The path-integral measure has been derived in [17] and in a slightly different language in [3] and in [38]. In [17] it was shown that one can find an acceptable integrand for the heterotic string given the holomorphic factorization of certain functional determinants. By the Belavin-Knizhnik theorem we know that the appropriate holomorphic square roots do, in fact, exist. Furthermore, it can be shown [41] that there is no obstruction to the holomorphic factorization in the supermoduli, and that after integration over the supermoduli the measure in [17]

\footnotetext{
${ }^{4}$ Two-dimensional supersymmetry requires that the NSR fermions and the background gravitino have the same spin structure

${ }^{5}$ This is very clearly explained in [31]
} 
can be cast in the form

$$
\begin{aligned}
Z= & \int_{F} \prod_{1}^{3 g-3} d t^{r} d t^{r *} \frac{\left|\operatorname{det}\left\langle S^{r} \mid T^{r \prime}\right\rangle\right|^{2}}{(\operatorname{det} \operatorname{Im} \Omega)^{5}} \frac{\operatorname{det} \Delta_{2}^{(+)}}{\operatorname{det}\left\langle S^{r} \mid S^{r \prime}\right\rangle}\left(\frac{\operatorname{det}^{\prime}-\nabla^{2}}{2 \pi \int \sqrt{g} \operatorname{det} \operatorname{Im} \Omega}\right)^{-5} \\
& \times \sum_{\alpha \beta \gamma} C_{\alpha \beta \gamma}\left(\operatorname{det}_{\beta} \nabla_{w}^{-1}\right)^{8}\left(\operatorname{det}_{\gamma} \nabla_{w}^{-1}\right)^{8}\left(\operatorname{det}_{\alpha} \nabla_{1}^{w}\right)^{5}\left(\frac{\operatorname{det}_{\alpha}\left\langle\chi_{i} \mid \chi_{j}\right\rangle}{\operatorname{det} \Delta_{1}^{(+)}}\right)^{1 / 2} \\
& \times \frac{1}{\operatorname{det}_{\alpha}\langle\chi \mid \chi\rangle} \int \operatorname{det}_{i, j} \chi_{i}\left(w_{j}\right)\left\langle S_{z \theta}\left(w_{1}\right) \ldots S_{z \theta}\left(w_{2 g-2}\right)\right\rangle .
\end{aligned}
$$

[We have chosen the case of $E_{8} \times E_{8}$ in (7.1).] Here we have represented Teichmüller space by a slice $\hat{g}(t)$ which has traceless symmetric tangent vectors $T^{r}$. The zero modes $S^{r}$ and $\chi_{i}$ form a basis for $\operatorname{ker} \nabla^{-4}$ and $\operatorname{ker} \nabla^{-3}$, respectively. The quantity $S_{z \theta}$ can be most easily expressed in the language of [3] as the ghost supercurrent: 6

$$
S_{z \theta}=\gamma^{\theta} b_{z z}+\beta_{z \theta} \nabla_{z}^{2} c^{z},
$$

where $c^{z} \gamma^{\theta}$ are the reparametrization and super-reparametrization ghosts and $b_{z z} \beta_{z \theta}$ are their conjugate fields. Thus the expectation value of the ghost currents can be expressed via Wick's theorem in terms of the Green's functions for the operators $\nabla_{z}^{1}$ and $\nabla_{z}^{2}$. Finally, one must choose the (anti)holomorphic square-root of $\operatorname{det} \Delta_{1}^{(+)}$, which exists, by the Belavin-Knizhnik theorem.

As is well-known, (7.1) has no local anomalies. Witten [30] has shown that the expression also has no global anomalies. His argument proceeds in two steps. First, it is shown that the terms with $\alpha=\beta=\gamma$ have no phase change under diffeomorphisms which preserve the spin structure $\alpha .{ }^{7}$ We cannot explicitly verify this because we do not know the modular dependence of the Rarita-Schwinger determinant. The second step of the proof in [30] proceeds by considering ratios of Dirac determinants. In particular, it is shown that (7.1) is modular invariant if

$$
\left(\frac{\operatorname{det}_{\alpha} \nabla_{z}^{-1}}{\operatorname{det}_{\beta} \nabla_{z}^{-1}}\right)^{4}
$$

is invariant under diffeomorphisms preserving spin structures $\alpha$ and $\beta$. We can explicitly verify this result since we know (7.2) as a function of the moduli, in particular, as a function of $\Omega$.

We can find the transformation of $\Omega$ under a nontrivial diffeomorphism as follows. Recall that if $\kappa_{i}=\left(a_{i}, b_{i}\right)$ is a canonical homology basis then $f_{*} \kappa_{i}=T_{i j} \kappa_{j}$, where

$$
T=\left(\begin{array}{ll}
D & C \\
B & A
\end{array}\right) \in S p(2 g, Z)
$$

\footnotetext{
${ }^{6}$ After an integration by parts

${ }^{7}$ Insertions of the ghost currents lead to Green's functions which at first sight vitiate the argument. However, the determinant over $\chi_{i}\left(w_{j}\right)$ suppresses the singular terms so that the correction factor is finite and does not need to be regularized. Since it needs no regularization, it should have no anomaly
} 
To preserve the normalization condition (3.6) we must redefine the basis of holomorphic differentials

$$
\tilde{\omega}_{i}=\omega_{j}(C \Omega+D)_{j i}^{-1} .
$$

Thus

$$
\Omega \rightarrow \widetilde{\Omega}_{i j}=\int_{\tilde{b}_{i}} \tilde{\omega}_{j}
$$

leads to the modular transformation

$$
\Omega \rightarrow \tilde{\Omega}=(A \Omega+B)(C \Omega+D)^{-1} .
$$

It can be shown [42] that the theta functions transform as

$$
\vartheta\left[\begin{array}{l}
\tilde{a} \\
\tilde{b}
\end{array}\right](\tilde{\Omega})=\varepsilon(\Lambda) e^{-i \pi \phi(a, b, \Omega)} \operatorname{det}(C \Omega+D)^{1 / 2} \vartheta\left[\begin{array}{l}
a \\
b
\end{array}\right](\Omega),
$$

where,

$$
\begin{gathered}
\Lambda=\left(\begin{array}{ll}
A & B \\
C & D
\end{array}\right), \\
{\left[\begin{array}{l}
\tilde{a} \\
\tilde{b}
\end{array}\right]=\left(\begin{array}{rr}
D & -C \\
-B & A
\end{array}\right)\left[\begin{array}{l}
a \\
b
\end{array}\right]+\frac{1}{2}\left[\begin{array}{l}
\left(C D^{t}\right)_{d} \\
\left(A B^{t}\right)_{d}
\end{array}\right],} \\
\phi(a, b, \Omega)=\left[a D^{t} B a+b C^{t} A b\right]-\left[2 a B^{t} C b+\left(a D^{t}-b C^{t}\right)\left(A B^{t}\right)_{d}\right] .
\end{gathered}
$$

The phase $\varepsilon$ is subtle. It is always an eighth root of unity and, if

$$
\Lambda=I_{2 g} \bmod 2 \text {, }
$$

then we have

$$
\varepsilon^{2}(\Lambda)=e^{\frac{i \pi}{2} \operatorname{tr}(D-1)} .
$$

In (7.4), the subscript $d$ means that we are taking the diagonal elements of the matrix to form a column vector. The above formula for the transformation of $\vartheta$ is valid for arbitrary real $g$-dimensional vectors $a$ and $b$. It can be easily verified for a set of generators of $\operatorname{Sp}(2 g, Z)$, although the extension to arbitrary symplectic transformations is more difficult.

We are now in a position to consider the transformation of (7.2). Let $\left[a_{1}, b_{1}\right]$ and $\left[a_{2}, b_{2}\right]$ be the half-integral characteristics corresponding to spin structures $\alpha$ and $\beta$ respectively. The modular transformation fixes spin structures $\alpha$ and $\beta$ if and only if for $i=1,2$

$$
\left[\begin{array}{c}
\tilde{a}_{i} \\
\tilde{b_{i}}
\end{array}\right]=\left[\begin{array}{l}
a_{i} \\
b_{i}
\end{array}\right] \bmod 1 .
$$

Using the explicit dependence of determinants, we would therefore like to show that

$$
\frac{\vartheta^{4}\left[\begin{array}{l}
\tilde{a}_{1} \\
\tilde{b}_{1}
\end{array}\right](\tilde{\Omega})}{\vartheta^{4}\left[\begin{array}{l}
\tilde{a}_{2} \\
\tilde{b}_{2}
\end{array}\right](\tilde{\Omega})}=\frac{\vartheta^{4}\left[\begin{array}{l}
a_{1} \\
b_{1}
\end{array}\right](\Omega)}{\vartheta^{4}\left[\begin{array}{l}
a_{2} \\
b_{2}
\end{array}\right](\Omega)}
$$


In the above expression we can shift the $a$ 's and $b$ 's by integral vectors, without changing the expression, because such a shift introduces a phase $e^{2 \pi i a \cdot n}$ for the theta function, where $n$ is an integral vector. This phase disappears when we raise the theta function to the fourth power. Using the modular transformation rule for the theta function, it is easy to see that the above equality holds provided

$$
e^{-i 4 \pi\left[\phi\left(a_{1}, b_{1}, \Omega\right)-\phi\left(a_{2}, b_{2}, \Omega\right)\right]}=1 .
$$

Since $a_{i}$ and $b_{i}$ are half-integral vectors, and $\Lambda$ is an integral matrix, it follows that a priori the above phase could be \pm 1 . To show that it is equal to 1 , one has to show that

$$
4\left(a_{1} D^{t} B a_{1}+b_{1} C^{t} A b_{1}-(1 \rightarrow 2)\right)=0 \bmod 2 .
$$

From now on for brevity, when we write an equality, we mean equality mod 2 . Note that in such expressions \pm signs play no role since $r=-r \bmod 2$ when $r$ is integral. Let us define integral vectors $c$ and $d$ by

$$
c=2 a_{1}-2 a_{2}, \quad d=2 b_{1}-2 b_{2} .
$$

The condition we will have to show becomes

$$
Q=c D^{t} B c+d C^{t} \mathrm{Ad}=0 .
$$

(We have used $D^{t} B=B^{t} D$ and $C^{t} A=A^{t} C$. This can be proved by writing the rightinverse of $A$ and demanding that it be a left-inverse.) The condition that the spin structures $\alpha$ and $\beta$ are preserved implies that (modulo two)

$$
\begin{aligned}
& (1+D) c+C d=0, \\
& B c+(1+A) d=0 .
\end{aligned}
$$

If we take the transpose of (7.7), multiply it by (7.8), and use $D^{t} A-B^{t} C=1$, we get

$$
Q+c\left(D^{t}+A\right) d+c B c+d C^{t} d=0 .
$$

Using (7.7) and (7.8) we see that

$$
0=2 c \cdot d=\left(\begin{array}{ll}
c & d
\end{array}\right)\left(\begin{array}{l}
d \\
c
\end{array}\right)=\left(\begin{array}{ll}
c & d
\end{array}\right)\left(\begin{array}{ll}
D^{t} & B^{t} \\
C^{t} & A^{t}
\end{array}\right)\left(\begin{array}{l}
d \\
c
\end{array}\right) .
$$

And so Eq. (7.9) simplifies to $Q=0$, the desired result. Thus, (7.1) is free of global anomalies. As noted in [30], four is the smallest power for which (7.2) is invariant. This is important in constraining variations on the heterotic string, as we will see below.

We now study the constants $C_{\alpha \beta \gamma}$ in (7.1). Naively, one might expect these constants to factorize for the sums over right- and left-movers, an impression which might be reinforced by the observation that the gauge fermions would then contribute a modular covariant sum:

$$
\sum_{a, b} \vartheta^{8}\left[\begin{array}{l}
a \\
b
\end{array}\right] \rightarrow \operatorname{det}(C \Omega+D)^{4} \sum_{a, b} \vartheta^{8}\left[\begin{array}{l}
a \\
b
\end{array}\right] .
$$

In fact, the standard $E_{8} \times E_{8}$ theory corresponds to this choice, but it is not the most general choice. The correct procedure, which was outlined in [30], involves modular orbits which we now describe. 
We have explained the one-to-one correspondence between spin structures and half-integral theta characteristics in Sect. 3. Using the transformation law (7.4) we can therefore find the orbit of a spin structure under the action of the modular group. As an example, we show that even and odd spin structures are separately permuted among themselves. The proof proceeds by induction. For $g=1$ the result is easily checked explicitly. In genus two we must use the nontrivial Dehn twist $D_{a_{1}^{-1} a_{2}}$ of Sect. 3a. The action of this twist on the half-integral characteristics is: ${ }^{8}$

$$
\begin{gathered}
{\left[\begin{array}{ll}
0 & 1 \\
a & b
\end{array}\right] \rightarrow\left[\begin{array}{ll}
0 & 1 \\
a & b
\end{array}\right] \quad\left[\begin{array}{ll}
1 & 0 \\
a & b
\end{array}\right] \rightarrow\left[\begin{array}{ll}
1 & 0 \\
a & b
\end{array}\right]} \\
{\left[\begin{array}{ll}
0 & 0 \\
a & b
\end{array}\right] \rightarrow\left[\begin{array}{cc}
0 & 0 \\
a+1 & b+1
\end{array}\right]}
\end{gathered}
$$

Using this transformation and the Dehn twists for the separate handles one can easily see that all the odd characcteristics of (3.29) can be transformed to

$$
\left[\begin{array}{ll}
0 & 1 \\
0 & 1
\end{array}\right]
$$

while all the even characteristics of (3.30) can be transformed to

$$
\left[\begin{array}{ll}
0 & 0 \\
0 & 0
\end{array}\right]
$$

We now assume that at $g-1$ loops all odd characteristics can be transformed to

$$
\left[\begin{array}{lll}
0 \ldots & 0 & 1 \\
0 \ldots & 0 & 1
\end{array}\right]
$$

while all even characteristics can be transformed to

$$
\left[\begin{array}{lll}
0 & \ldots & 0 \\
0 & \ldots & 0
\end{array}\right]
$$

Consider any even characteristic at $g$ loops. The first $g-1$ columns define an even or odd spin structure depending on whether the final column is even or odd. In the case that both are even we can use the inductive hypothesis and Dehn twists on the $g^{\text {th }}$ handle to bring the characteristic to the form (7.12). In the case that both are odd we again use the inductive hypothesis to transform the $g$-loop characteristic to

$$
\left[\begin{array}{llll}
0 \ldots 0 & 1 & 1 \\
0 \ldots 0 & 1 & 1
\end{array}\right]
$$

Applying the transformation (7.10) to the last two handles and using two further torus-Dehn-twists, we can finally bring the $g$-loop characteristic to the form (7.12). A similar argument for an arbitrary odd characteristic at $g$-loops completes the inductive step. Thus, the set of spin structures decomposes into two distinct modular orbits.

\footnotetext{
${ }^{8}$ For notational simplicity we have multiplied all the half-integral characteristics by two. They are therefore specified by zeros and ones, modulo two
} 
When we consider more than one independent spin structure, the modular orbits become more intricate. For example, in the $E_{8} \times E_{8}$ heterotic string we must consider triplets of spin structures $\alpha \beta \gamma$. Each such triplet has an associated modular orbit $\mathcal{O}$ obtained by simultaneously transforming all three spin structures. Denote by $S[\alpha \beta \gamma]$ the contribution of the functional determinants in (7.1) for the triplet of spin structures $\alpha \beta \gamma$. It is easy to check that if $S$ is invariant under diffeomorphisms preserving the triplet of spin structures, then the sum

$$
\sum_{[\alpha \beta \gamma] \in \mathcal{O}} S[\alpha \beta \gamma]
$$

is modular invariant. This leaves an ambiguity in the definition of the amplitude (7.1), for if we write the integrand as

$$
I_{g}=\sum_{\text {orbits } \mathcal{O}} C(\mathcal{O}) \sum_{[\alpha \beta \gamma] \in \mathcal{O}} S[\alpha \beta \gamma],
$$

the coefficients $C$ are not fixed by absence of global anomalies on the world sheet.

The importance of modular invariance and factorization in relating different terms in the path-integral measure for superstrings has been pointed out in [31]. Similar ideas have been applied in the context of orbifolds in [43] and in determining inequivalent phases for heterotic strings in [32]. In particular these ideas have led to new heterotic strings $[44,31,32]$. We will now discuss some aspects of the new theories using the transformation law (7.3), (7.4) for the theta characteristics. We will only investigate the possible modifications of the $O(32)$ and $E_{8} \times E_{8}$ strings for simplicity. (One can also consider breaking up the sets of fermions further into sets in eight, or some multiples of eight, adding up to 32 fermions. This is the furthest one can go: since four is the smallest power for which (7.2) is invariant, allowing smaller sets of fermions would lead to a measure with global anomalies [30].)

An amplitude factorizes if, in the corner of Teichmüller space corresponding to the degeneration of a Riemann surface illustrated in Fig. $6^{*}$ (that is, when the handles are pulled apart), the integrand factorizes into a product of $g_{1}$ - and $g_{2}$-loop amplitudes: $I_{g} \rightarrow I_{g_{1}} I_{g_{2}}$. In this limit the period matrix becomes [14]

$$
\Omega \rightarrow\left(\begin{array}{cc}
\Omega_{1} & 0 \\
0 & \Omega_{2}
\end{array}\right)
$$

so the theta functions also factorize into $g_{1}-$ and $g_{2}$-loop theta functions:

$$
\vartheta\left[\begin{array}{l}
a \\
b
\end{array}\right](0 \mid \Omega) \rightarrow \vartheta\left[\begin{array}{l}
a_{1} \\
b_{1}
\end{array}\right]\left(0 \mid \Omega_{1}\right) \vartheta\left[\begin{array}{l}
a_{2} \\
b_{2}
\end{array}\right]\left(0 \mid \Omega_{2}\right) .
$$

We will assume that the supersymmetry ghost determinant and the supermoduli correction (which depend on spin structure) similarly factorize so that

$$
I_{g_{1}+g_{2}} \rightarrow \sum_{\text {orbits } \mathcal{O}} C_{g_{1}+g_{2}}(\mathcal{O}) \sum S\left[\alpha_{1} \beta_{1} \gamma_{1}\right] S\left[\alpha_{2} \beta_{2} \gamma_{2}\right],
$$

Fig. 6

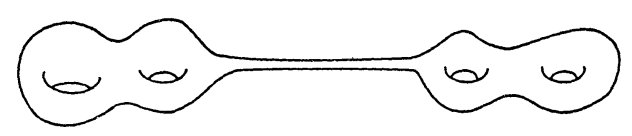


where $\left[\alpha_{i} \beta_{i} \gamma_{i}\right]$ are any spin structures at genus $g_{i}$ which combine to give a spin structure in orbit $\mathcal{O}$ at genus $g$. By our assumption that the $S$ 's factorize, the choice $C=1$ is consistent with factorization of the amplitude, but this is not the unique choice. In general the coefficients must satisfy

$$
C_{g_{1}+g_{2}}(\mathcal{O}[\alpha \beta \gamma])=C_{g_{1}}\left(\mathcal{O}\left[\alpha_{1} \beta_{1} \gamma_{1}\right]\right) C_{g_{2}}\left(\mathcal{O}\left[\alpha_{2} \beta_{2} \gamma_{2}\right]\right) \text {, }
$$

where $\mathcal{O}[\alpha \beta \gamma]$ denotes the modular orbit of a triplet of spin structures. Note that if two choices of coefficients satisfy (7.16) then so does their product. Thus the solutions of (7.16) form a group. The condition (7.16) is nontrivial because two triplets of spin structures, $\alpha \beta \gamma$ and $\mu \nu \varrho$, in the same modular orbit at $\left(g_{1}+g_{2}\right)$-loops can factorize into products of triplets of spin structures

$$
[\alpha \beta \gamma] \rightarrow\left[\alpha_{1} \beta_{1} \gamma_{1}\right]\left[\alpha_{2} \beta_{2} \gamma_{2}\right], \quad[\mu \nu \varrho] \rightarrow\left[\mu_{1} v_{1} \varrho_{1}\right]\left[\mu_{2} v_{2} \varrho_{2}\right]
$$

in different modular orbits at $g_{1}$ - or $g_{2}$-loops. Thus we must have

$$
C\left(\mathcal{O}\left[\alpha_{1} \beta_{1} \gamma_{1}\right]\right) C\left(\mathcal{O}\left[\alpha_{2} \beta_{2} \gamma_{2}\right]\right)=C\left(\mathcal{O}\left[\mu_{1} v_{1} \varrho_{1}\right]\right) C\left(\mathcal{O}\left[\mu_{2} v_{2} \varrho_{2}\right]\right)
$$

for all pairs of triplets factorizing from $\left(g_{1}+g_{2}\right)$-loop triplets which have the same modular orbit $\mathcal{O}$. From (7.16) we see that the $g$-loop coefficients can be written as products of one-loop coefficients. Since $\Omega(\Sigma)$ is generated by the Dehn twists about the curves in Fig. 4 we see that the only nontrivial conditions from (7.18) come from taking $g_{1}=g_{2}=1$, and if these are satisfied then (7.18) holds for all $g_{1}, g_{2}$, and all triplets of spin structures.

There are always some trivial solutions of (7.18) which we now describe. Consider either the first, second, or third set of fermions. It is easy to check that if we take $C=+1$ when this set has an even spin structure and $C=-1$ when the set has an odd spin structure, then (7.18) is satisfied. These solutions form the group $Z_{2}$ $\times Z_{2}$ for the $O$ (32) string and $Z_{2} \times Z_{2} \times Z_{2}$ for the $E_{8} \times E_{8}$ string. By comparing the modified signs in the path integral with the corresponding expressions in the operator formalism one finds that these solutions correspond to physically irrelevant redefinitions of the chiralities of the massless ground state spinors in the spectrum of the theory.

We now search for nontrivial solutions of (7.18) in the specific example of the $O(32)$ string. In this case the modular orbits are generated by doublets of spin structures. There are five distinct one-loop modular orbits: Either both spin structures are even and identical, or both are even and distinct, or one is odd and the other is even, or both are odd. These may be denoted by ee ee $e^{\prime}$ eo oe oo. We may choose $C(e e)=1$ by convention. We can see that $C\left(e e^{\prime}\right)=1$ by considering the following example. The genus two doublet of spin structure with characteristics

$$
\left[\begin{array}{ll}
0 & 0 \\
0 & 0
\end{array}\right]_{1}\left[\begin{array}{ll}
0 & 1 \\
0 & 0
\end{array}\right]_{2}
$$

factorizes into the product of doublets of one-loop spin structures:

$$
\left(\left[\begin{array}{l}
0 \\
0
\end{array}\right]_{1}\left[\begin{array}{l}
0 \\
0
\end{array}\right]_{2}\right)\left(\left[\begin{array}{l}
0 \\
0
\end{array}\right]_{1}\left[\begin{array}{l}
1 \\
0
\end{array}\right]_{2}\right)
$$


which has coefficient $C\left(e e^{\prime}\right)$ in the product of one-loop amplitudes (since $C(e e)=1)$. On the other hand the doublet of spin structures with characteristics

$$
\left[\begin{array}{ll}
0 & 0 \\
1 & 1
\end{array}\right]_{1}\left[\begin{array}{ll}
0 & 1 \\
0 & 0
\end{array}\right]_{2}
$$

factorizes into the product of doublets:

$$
\left(\left[\begin{array}{l}
0 \\
1
\end{array}\right]_{1}\left[\begin{array}{l}
0 \\
0
\end{array}\right]_{2}\right)\left(\left[\begin{array}{l}
0 \\
1
\end{array}\right]_{1}\left[\begin{array}{l}
1 \\
0
\end{array}\right]_{2}\right)
$$

which has coefficient $C\left(e e^{\prime}\right)^{2}$. Since the orbits of (7.19) and (7.21) are related by the Dehn twist $D_{a_{\overline{1}}^{1} a_{2}}$ we must have $C\left(e e^{\prime}\right)^{2}=C\left(e e^{\prime}\right)$, which means $C\left(e e^{\prime}\right)=0$ or 1 . The first choice leads to a theory with tachyons [31], so we investigate the second choice. We then have effectively only four distinct modular orbits corresponding to whether the members of the doublet of spin structures are even or odd. Since a spin structure with the characteristics $\left[\begin{array}{l}a \\ b\end{array}\right]$ is even or odd depending on whether $a b=0$ or $1 \bmod 2$, we can consider $C$ as a function on $Z_{2} \times Z_{2}$. Generalizing the above example of (7.19)-(7.22) to the genus two orbit of

$$
\left[\begin{array}{ll}
a & a^{\prime} \\
b & b^{\prime}
\end{array}\right]_{1}\left[\begin{array}{ll}
c & c^{\prime} \\
d & d^{\prime}
\end{array}\right]_{2}
$$

we find the condition

$$
C(a b, c d) C\left(a^{\prime} b^{\prime}, c^{\prime} d^{\prime}\right)=C\left(a b+a a^{\prime}, c d+c c^{\prime}\right) C\left(a^{\prime} b^{\prime}+a a^{\prime}, c^{\prime} d^{\prime}+c c^{\prime}\right)
$$

which implies

$$
C(0,1)^{2}=C(1,0)^{2}=1, \quad C(1,1)=C(0,1) C(1,0),
$$

which corresponds to the trivial solutions found above. A similar but more tedious analysis of the $E_{8} \times E_{8}$ case shows that the solutions form the group $Z_{2} \times Z_{2} \times Z_{2}$ $\times Z_{2}$. The first three $Z_{2}^{\prime} s$ correspond to the trivial solutions discussed above, but the fourth $Z_{2}$ is nontrivial, and leads to a heterotic string with $O(16) \times O(16)$ gauge symmetry $[44,32]$.

In general we can start with all spin structures identified $[31,32]$ and take the quotient by various groups to get the other theories. If we take the quotient with groups which are direct products of $Z_{2}$, we can get sums over up to five independent spin structures. For example, using a single $Z_{2}$ we can get the $O(32)$ heterotic string or a tachyonic $O(16) \times E_{8}$ string $[44,31]$, where the latter theory is obtained by identifying the spin structure of the right moving spacetime fermions with the spin structure of eight of the left moving gauge fermions. It seems that in all the known examples identification of the spin structures of some left and right movers leads to a theory with tachyons.

\section{Conclusions}

Much work remains to be done. We have seen that theta functions are useful for understanding spin one-half particles. Can the ghost determinants and zero modes 
also be usefully expressed in theta functions? We have also seen that some pieces of the string path integral extend as functions of the Siegel upper half plane. Can the entire string integrand be expressed as a function on this space [45]? If so, what are the constraints of symplectic modular invariance on physical theories? We have investigated one simple aspect of bosonization on higher genus surfaces, but a full treatment is still lacking.

The naive expectation that the determinant of the spin- $\frac{3}{2}$ operator should be given in its spin-structure-dependence by a theta function is misguided, as explained in Sect. 5. Furthermore, although there are identities (known as the Riemann identities) which are of the form $\sum C_{\alpha} \vartheta^{4}=0$ [13], they are not (formally) modular covariant. A direct proof of the vanishing of the cosmological constant will involve more sophisticated identities, and might point the way to new Schottky relations on the period matrix. We simply note here that a direct approach is likely to encounter subtleties, since there must be delicate cancellations between the contributions of the various spin structures. Indeed, merely changing a few of the coefficients $C_{\alpha \beta \gamma}$ leads to the $O(16) \times O(16)$ heterotic string which is modular invariant and unitary, and yet has a finite nonzero cosmological constant.

Acknowledgements. We would like to thank P. Nelson for suggesting many improvements of a preliminary manuscript and for many conversations on strings and Riemann surfaces. We would also like to thank Stephen Della Pietra, Vincent Della Pietra, D. Freed, P. Ginsparg, D. Mumford, J. Polchinski, and I. Singer for much help throughout the course of this work. This work was supported in part by NSF contract PHY-82-15249. G.M. and C.V. are also supported by the Harvard Society of Fellows, and L. A.-G. by the A.P. Sloan Foundation.

Similar results on the dependence of the Dirac determinant on spin structure have also been obtained by D. Freed from the viewpoint of the geometry of index bundles and by J. Fay using the Szegö kernel.

\section{References}

1. Schwarz, J.: Superstrings: The first fifteen years, Vol. 5, I, II. New York: World Scientific 1985

2. Polyakov, A.M.: Quantum geometry of bosonic strings. Phys. Lett. 103B, 207 (1981); Quantum geometry of fermionic strings. Phys. Lett. 103B, 211 (1981)

3. Friedan, D., Martinec, E., Shenker, S.: Conformal invariance, supersymmetry, and strings. Princeton preprint

4. Martinec, E.: Nonrenormalization theorems and fermionic string finiteness. Princeton preprint

5. Gross, D.J., Harvey, J.A., Martinec, E., Rohm, R.: Phys. Rev. Lett. 54, 502 (1985); Nucl. Phys. B256, 253 (1985), and Nucl. Phys. B (to appear)

6. Dixon, L., Harvey, J., Vafa, C., Witten, E.: Strings on orbifolds I. Nucl. Phys. B261, 651 (1985). Strings on orbifolds II. Princeton preprint

7. Farkas, H.M., Kra, I.: Riemann surfaces. Berlin, Heidelberg, New York: Springer 1980

8. See, for example J. Birman in W.J. Harvey (ed.), Discrete groups and automorphic functions. New York: Academic Press 1977; L. Greenberg (ed.), Discontinuous groups and Riemann surfaces, Princeton, NJ: Princeton University Press 1974

9. Magnus, Karras, Solitar: Combinatorial group theory. New York: Interscience 1966

10. Milnor, J.W., Stasheff, J.D.: Characteristic classes. Ann. Math. Studies, Vol. 76. Princeton, NJ: Princeton University Press 1974 
11. Griffiths, P., Harris, J.: Principles of algebraic geometry. New York: Wiley 1978

12. Gunning, R.: Introduction tc Riemann surfaces princeton Math. Notes. Princeton, NJ: Princeton University Press 1966

13. Mumford, D.: Tata lectures on theta, Vols. I, II. Boston: Birkhäuser 1983

14. Fay, J.: Theta functions on Riemann surfaces Springer notes in math., Vol. 352. Berlin, Heidelberg, New York: Springer 1973

15. Quillen, D.: Determinants of Cauchy-Riemann operators on Riemann surfaces. Funkts. Anal. Prilozh. 19, 37 (1985)

16. Alvarez, O.: Conformal anomalies and the index theorem. Berkeley preprint

17. Moore, G., Nelson, P., Polchinski, J.: Strings and supermoduli. Phys. Lett. 169B,47 (1986)

18. Belavin, A.A., Knizhnik, V.G.: Algebraic geometry and the geometry of quantum strings. Landau Institute preprint; See also, Catenacci, R., Cornalba, M., Martellini, M., Reina, C.: Algebraic geometry and path integrals for closed strings; Bost, J.B., Jolicoeur, J.: A holomorphy property and critical dimension in string theory from an index theorem. Saclay $\mathrm{PhT} / 86-28$

19. Alvarez-Gaumé, L., Witten, E.: Gravitational anomalies. Nucl. Phys. B234, 269 (1984)

20. Actually, this particular example is well-known to physicists. See, for example, Jackiw, R.: Topological methods in field theory. Les Houches lectures 1983. What is new here is the better geometrical understanding in terms of holomorphic line bundles, and the idea that holomorphy is a powerful tool for understanding determinants

21. The generalization of Quillen's theorem has been independently derived by Belavin and Knizhnik. We thank Stephen Della Pietra for pointing out an error in an earlier version of Eq. (4.15). We also thank Phil Nelson and Joe Polchinski for discussions on the application of Eq. (4.15) to holomorphic factorization on moduli space, and on the important difference between Eq. (4.15) and Eq. (4.16)

22. Bismut, J.-M., Freed, D.S.: Geometry of elliptic families: Anomalies and determinants. M.I.T. preprint; The analysis of elliptic families: Metrics and connections on determinant bundles. Commun. Math. Phys. (in press); The analysis of elliptic families: Dirac operators, eta invariants, and the holonomy theorem. Commun. Math. Phys. (in press)

23. This can also be understood from the point of view of Wolpert, S.: On obtaining a positive line bundle from the Weil-Petersson class. Am. J. Math. 107, 1485 (1985)

24. Bers, L.: Finite dimensional Teichmüller spaces and generalizations. Bull. Am. Math. Soc. 5, 131 (1981)

25. Friedan, D.: Introduction to Polyakov's string theory. In: Zuber, J.-B., Stora, R. (eds.). Les Houches 1982. Recent advances in field theory and statistical mechanics. Amsterdam: Elsevier 1984

26. We owe this observation to Phil Nelson

27. Atiyah, M.: Riemann surfaces and spin structures. Ann. Sci. Ec. Norm. Super. 4 (1971)

28. In fact, more is true: the number of zero modes is given by the multiplicity of the zero of $\vartheta$. See [14] and Mumford, D.: Theta characteristics of an algebraic curve. Ann. Sci. Éc. Norm. Supér. 4, 24 (1971)

29. Hitchin, N.: Harmonic spinors. Adv. Math. 14, 1-55 (1974)

30. Witten, E.: Global anomalies in string theory. In: Geometry anomalies topology. Bardeen, W.A., White, A.R. (eds.). New York: World Scientific 1985

31. Seiberg, N., Witten, E.: Spin structures in string theory. Princeton preprint

32. Alvarez-Gaumé, L., Ginsparg, P., Moore, G., Vafa, C.: An $O(16) \times O(16)$ heterotic string. Harvard preprint HUTP-86/AO13

33. For a pedagogical treatment and further references to the literature see Nelson, P., Moore, G.: Heterotic geometry. Harvard preprint HUTP-86/A014

34. Alvarez, O.: Theory of strings with boundary: Topology, fluctuations, geometry. Nucl. Phys. B216, 125 (1983)

35. Polchinski, J.: Evaluation of the one loop string path integral. Commun. Math. Phys. 104, 37 (1986) 
36. Moore, G., Nelson, P.: Measure for moduli: The Polyakov string has no nonlocal anomalies. Nucl. Phys. B266, 58 (1986)

37. D'Hoker, E., Phong, D.: Multiloop amplitudes for the bosonic string. Nucl. Phys. B 269, 205 (1986)

38. D'Hoker, E., Phong, D.: Loop amplitudes for the fermionic string. CU-TP-340

39. Shapiro, J.: Loop graph in the dual-tube model. Phys. Rev. D5, 1945 (1972); Rohm, R.: Spontaneous supersymmetry breaking in supersymmetric string theories. Nucl. Phys. B237, 553 (1984)

40. Gliozzi, F., Scherk, J., Olive, D.: Supersymmetry, supergravity theories and the dual spinor model. Nucl. Phys. B122, 253 (1977)

41. In preparation

42. Igusa, J.: Theta functions. Berlin, Heidelberg, New York: Springer 1972

43. Vafa, C.: Modular invariance and discrete torsion on orbifolds

44. Dixon, L., Harvey, J.: String theories in ten dimensions without spacetime supersymmetry. Princeton preprint

45. After this work was completed we received a preprint in which this question is answered in the affirmative. See Manin, Yu.I.: The partition function of the Polyakov string can be expressed in terms of theta functions. Phys. Lett. (submitted)

Communicated by A. Jaffe

Received April 4, 1986 